\title{
Pseudodifferential analysis on manifolds with boundary - a comparison of b-calculus and cone algebra
}

\author{
R. Lauter and J. Seiler
}

\begin{abstract}
We establish a relation between two different approaches to a complete pseudodifferential analysis of totally characteristic or Fuchs type operators on compact manifolds with boundary respectively conical singularities: Melrose's (overblown) b-calculus and Schulze's cone algebra. Though quite different in their definition, we show that these two pseudodifferential calculi basically contain the same operators.
\end{abstract}

\section{Introduction}

We compare two different approaches to a pseudodifferential analysis of totally characteristic or Fuchs type operators on compact manifolds with boundary. Recall that locally differential operators in this setting are of the form

$$
x^{-m} \sum_{k+|\alpha|=0}^{m} a_{k \alpha}(x, y)\left(x \partial_{x}\right)^{k} \partial_{y}^{\alpha}
$$

with $a_{k \alpha} \in \mathcal{C}^{\infty}\left(\overline{\mathbb{R}}_{+} \times \mathbb{R}^{n-1}\right)$. Here, $(x, y) \in \overline{\mathbb{R}}_{+} \times \mathbb{R}^{n-1}$ are local coordinates near the boundary; the weight $x^{-m}$ sometimes can be omitted. In this context, it is of interest to characterize the Fredholm operators (in an appropriate scale of weighted Sobolev spaces) and the solutions to elliptic equations. A natural way to treat these problems is to construct a pseudodifferential calculus that includes the parametrices of Fredholm operators, where a parametrix is an inverse up to remainders in a 'small' residual class within the calculus. Such pseudodifferential calculi for totally characteristic operators together with a complete symbolic structure have been developed independently by Melrose [28], [31], Plamenevskij [42], [43], Rempel, Schulze [45], Schulze [55], [57], and Unterberger [65]. Let us mention that Fuchs

1991 Mathematics Subject Classification. Primary 58G15; Secondary 47G30.

Key words and phrases. pseudodifferential analysis, manifolds with boundary, manifolds with conical singularities, b-calculus, cone algebra.

The first author was supported by a scholarship of the German Academic Exchange Service (DAAD) within the Hochschulsonderprogramm III von Bund und Ländern. 
type operators were also treated by Brüning [1], Brüning, Seeley [2], Cheeger [3], Lesch [21], and many others. In this paper, however, we concentrate on the bcalculus of Melrose and the cone algebra of Schulze. Though the presentation of these two calculi is quite different, in fact, these classes of operators are almost the same. Before making this more precise, let us give a brief description of the main features of the two calculi.

In [28], Melrose observed that important parts of the theory of totally characteristic operators on a compact manifold with boundary can be understood geometrically. In fact, the Schwartz kernels of totally characteristic or briefly bpseudodifferential operators can be characterized on a compact manifold with corners $X_{b}^{2}$, that is obtained from the product manifold $X^{2}$ by blowing up the corner $(\partial X)^{2}$. To be more precise, as in the closed case [10], the kernels of bpseudodifferential operators in the small calculus are conormal to the (lifted) diagonal $\Delta_{b}$, and vanish, in addition, to arbitrary order on all boundary faces of $X_{b}^{2}$ not intersecting the submanifold $\Delta_{b}$. The normal bundle $N \Delta_{b}$ of this $b$-diagonal can canonically be identified with the b-tangent bundle ${ }^{b} T X$, a smooth vector bundle whose space of smooth sections coincides with the Lie algebra $\mathcal{V}_{b}(Z)$ of all vector fields on $X$ that are tangent to the boundary, i.e., roughly speaking, the space of totally characteristic or b-differential operators of order 1. Consequently, the b-tangent bundle plays the same role in the b-calculus as the usual tangent bundle in the ordinary pseudodifferential calculus on closed manifolds. In particular, its dual ${ }^{b} T^{*} X$, the $b$-cotangent bundle, carries the homogeneous principal symbol for b-pseudodifferential operators. As explained in [31], the b-calculus provides an appropriate setting for understanding the Atiyah-Patodi-Singer index theorem, and, in particular, the appearance of the eta-invariant.

Though the small calculus suffices to invert "elliptic" b-pseudodifferential operators up to operators of order $-\infty$, it is not sufficient to get compact remainders because of non trivial boundary contributions. To obtain compact or even finitedimensional remainders one has to admit operators of order $-\infty$ with a more general asymptotic behavior at the faces of $X_{b}^{2}$ (and also $X^{2}$ ). This leads to the full calculus $\Psi_{b, c l}^{*, \mathcal{E}}\left(X,{ }^{b} \Omega^{\frac{1}{2}}\right)$ of b-pseudodifferential operators. Here, $\mathcal{E}$ is an index family describing the form of the asymptotic behavior. The definitions and basic results are given in Section 4; for more details we refer to [31] or [36].

Note that for compact remainders, i.e. for a Fredholm theory we do not really need the full asymptotic information contained in $\mathcal{E}$, but only a certain degree of vanishing at the faces. However, this calculus with bounds [31, Section 5.16] is not the main topic of this paper.

The definition of the b-calculus extends naturally to the more general case of manifolds with corners [22], [39]. The corresponding algebras of operators of order zero were investigated in $[\mathbf{1 7}],[\mathbf{1 9}]$, and $[\mathbf{3 7}]$ from a functional analytic point of view.

It is worth pointing out that the b-caluclus fits into the setting of and is, in fact, the prototype of a boundary fibration structure where Lie algebras of vector 
fields are used to characterize degenerate behavior on manifolds with boundary or corners [29], [35]. For the construction of the corresponding pseudodifferential calculi we refer the reader for instance to $[\mathbf{2 0}],[\mathbf{2 5}],[\mathbf{2 6}],[\mathbf{3 2}],[\mathbf{3 3}],[\mathbf{3 4}],[\mathbf{3 8}]$.

The passage from a manifold with conical singularities to a manifold with boundary by blowing-up, naturally leads to the consideration of Fuchs-type operators. An easy example are differential operators on $\mathbb{R}^{n}$ that have the structure (1.1) if expressed in polar coordinates. Also geometric operators like the LaplaceBeltrami operator on a manifold with boundary with respect to a conical metric are of this form (with $m=2$ ).

In [13] Kondrat'ev observed that the solutions to elliptic differential boundary value problems in conical domains have specific asymptotics near the vertex, which are related to the eigenvalues of resulting boundary value problems on the cross-section. Pointing out such a global behavior along the base of the cone, the operators in the cone algebra are described near the singularity in terms of Mellin pseudodifferential operators with operator-valued symbols. For example, the Mellin symbol of a Fuchs-type differential operator is a polynomial in $z \in \mathbb{C}$ and coefficients that are functions with values in the differential operators on the cross-section. To describe the parametrices, this holomorphic structure is not sufficient. General operators of the cone algebra are built upon operator-valued symbols that extend in the covariable meromorphically to the complex plane. It is this meromorphic structure that induces the typical asymptotic behavior near the singularity of the solutions to elliptic equations. As a matter of fact, such symbols can be split into a holomorphic part of full order and a meromorphic part of order $-\infty$. Therefore, the asymptotic information is carried by smoothing cone operators. If interested only in parametrices modulo compact operators, it suffices to consider smoothing symbols that only extend holomorphically to small vertical strips in the plane, cf. for example [6], [61]. The width of these strips corresponds precisely (after Mellin transform) to the degree of vanishing at the faces of the kernels in the b-calculus with bounds mentioned above.

Let us mention that the aspect of operator-valued symbols is interesting for various reasons. In particular, it yields an iterative approach to manifolds with higher singularities (such as manifolds with edges [50], [51], [54], [61], and manifolds with corners [56], [59]), where the operators have symbols with values in operator algebras on less singular spaces. For instance, a manifold with corner is (locally) a cone over a base, which itself has conical singularities. A general method of iterating pseudodifferential calculi is discussed in [60]. The use of operator-valued symbols also allows to obtain analytic index formulas in the spirit of Fedosov, cf. $[\mathbf{6}],[7]$. Note also that in $[\mathbf{4 0}],[\mathbf{5 3}]$ a characterization of the stable homotopy classes of elliptic leading symbols is given (in the case of conical singularities).

It has been shown in $[\mathbf{1 6}]$ that the $C^{*}$-algebras generated by the operators of order 0 in the small b-calculus coincides with that generated by the cone algebra. Before, these algebras were studied independently in [15], [23], and [37]. In this paper, we analyze the relation between the two calculi for arbitray orders more precisely. As a first result, we show that the small (overblown) b-calculus coincides 
(up to a weight factor) with the space of cone operators having holomorphic Mellin symbols (Theorem 5.4). The comparison of the full b-calculus with the cone algebra therefore reduces to operators of order $-\infty$. In fact, the pattern of poles together with multiplicities is reflected in the asymptotic behavior of the kernels near the boundary faces of $X_{b}^{2}$, and vice versa (Theorem 5.8, Proposition 5.10, and Corollary 5.15). As a consequence (Theorem 5.17) we obtain for manifolds $X$ with connected boundary

$$
\mathfrak{C}^{m}\left(X,{ }^{b} \Omega^{\frac{1}{2}},(\gamma, \gamma-m, \infty)\right) \subset \bigcup_{\mathcal{E}} \varrho^{-m} \Psi_{b}^{m, \mathcal{E}}\left(X,{ }^{b} \Omega^{\frac{1}{2}}\right)=\mathfrak{C}_{w}^{m}\left(X,{ }^{b} \Omega^{\frac{1}{2}},(\gamma, \gamma-m, \infty)\right),
$$

where $\mathcal{E}$ describes the asymptotics of the kernels near the various faces of $X_{b}^{2}$, and the union is taken over all $\mathcal{E}$ satisfying a certain compatibility relation with the weight data $(\gamma, \gamma-m)$, cf. (5.12). Moreover, by the subscript $w$ we denote a slightly enlarged, 'weak' version of the cone algebra, introduced in Section 3.

An essential step in the proof of (1.2) is explaining the precise relationship between the spaces of polyhomogeneous conormal functions (Definition 4.3) and the spaces with (weak) discrete asymptotics (Definition 3.4 ) that are used in bcalculus resp. cone algebra to describe the asymptotic behavior at the boundary faces. Results for $X$ resp. $X^{2}$ can be found in Corollary 5.6 resp. Theorem 5.8.

We want to point out that the cone algebra can be shown [52] to be spectrally invariant. This becomes useful, for instance, in characterizing parametrices for higher singularities, since then ellipticity is just the invertibility of certain operatorfunctions taking values in calculi of the lower levels. Spectral invariance for the b-calculus holds for a slightly restricted subalgebra of the full-calculus.

However, the algebra of operators of order 0 either in the full b-calculus or in the cone algebra is quite complicated from the point of view of topological algebras - this is discussed in [16] in detail. In particular, it is not known whether these algebras though spectrally invariant are closed under holomorphic functional calculus. Nevertheless, it is reasonable to expect $f(a)$ within the same class as $a$ provided $f$ is not only holomorphic near the $L^{2}$-spectrum of $a$ but also near regions corresponding to the boundary symbol (for instance entire functions). This complicated behavior of totally characteristic operators was the reason for constructing in [18] $\Psi^{*}$-algebras of totally characteristic operators containing and sharing important $\mathcal{C}^{\infty}$-properties with b-calculus and cone-algebra. Recall that $\Psi^{*}$-algebras introduced by Gramsch in $[\mathbf{8}]$ are not only closed under holomorphic functional calculus in the usual sense but also an appropriate notion for understanding smooth and microlocal phenomena in pseudodifferential analysis from a functional analytic point of view - for general techniques that can be used in this context we refer the reader to $[\mathbf{9}],[\mathbf{1 4}]$, or $[\mathbf{2 4}]$.

The main results of this paper are stated and proved in Section 5. In Section 2 we recall some basic facts on manifolds with corners and conical singularities, we give a review on the cone algebra in Section 3, and a summary of the b-calculus in Section 4. 
Acknowledgements: We thank R. B. Melrose, E. Schrohe, and B.-W. Schulze for valuable discussion and support. The first named author wishes to thank the Massachusetts Institute of Technology, where parts of the paper were written for the invitation and the warm hospitality.

\section{Preliminaries}

2.1. Manifolds with corners. The presentation of this subsection is essentially extracted from [31]. For more details on manifolds with corner we refer the reader to $[\mathbf{5}],[\mathbf{2 7}],[\mathbf{3 0}]$, or $[\mathbf{3 5}]$.

Recall [31, Section 2] that a manifold with corners of dimension $n$ is a (connected) $n$-dimensional, topological manifold $Z$ with boundary together with a homeomorphism $i: Z \stackrel{\approx}{\longrightarrow} \widetilde{Z} \subseteq M_{Z}$ into a smooth $n$-dimensional manifold $M_{Z}$ without boundary such that there exists a finite family $\varrho_{j} \in \mathcal{C}^{\infty}\left(M_{Z}\right), j \in J$, of smooth functions satisfying

1. $i(Z)=\widetilde{Z}=\bigcap_{j \in J}\left\{\varrho_{j} \geq 0\right\}$.

2. $\left.d \varrho_{j}\right|_{p} \in T_{p}^{*} M_{Z}, j \in J_{p}:=\left\{j \in J: \varrho_{j}(p)=0\right\}$ are linearly independent for all $p \in \tilde{Z}$.

The $\mathcal{C}^{\infty}$-structure on $Z$ is given by $\mathcal{C}^{\infty}(Z):=i^{*} \mathcal{C}^{\infty}\left(M_{Z}\right)$. Such a manifold $M_{Z}$ is also said to be an extension of $Z$, and it is straightforward to check that the $\mathcal{C}^{\infty}$-structure on $Z$ is independent of the extension $M_{Z}$. A manifold with boundary is a manifold with corners where $J$ can be taken to have one element. The usual objects of differential topology are defined by pulling-back the corresponding ones on $M_{Z}$.

A subset $F \subseteq Z$ corresponding under $i$ to a component of $\cap_{j \in I}\left\{\varrho_{j}=0\right\}$ for some $I \subseteq J$ with $|I|=k$ is called a (boundary) face of codimension $k$, and we write $\mathcal{F}_{k}(Z)$ for the family of all boundary faces of codimension $k$.

Let us call a smooth function $\varrho_{F}: Z \longrightarrow \overline{\mathbb{R}}_{+}$a defining function for the boundary hyperface $F \in \mathcal{F}_{1}(Z)$ provided $F=\left\{\varrho_{F}=0\right\}$ and $d \varrho_{F} \neq 0$ at $F$. Of course, a defining function for $F$ is not uniquely determined. However, for any two defining functions $\varrho_{F}, \varrho_{F}^{\prime}$ of $F$ there exists $0<a \in \mathcal{C}^{\infty}(Z)$ with $\varrho_{F}^{\prime}=a \varrho_{F}$.

Note that there is also an intrinsic definition of a manifold with corners built on local coordinates modeled on the spaces $\mathbb{R}_{k}^{n}:=\overline{\mathbb{R}}_{+}^{k} \times \mathbb{R}^{n-k}$ and the additional requirement that all boundary faces are embedded - for details we refer to [35].

Naturally associated to a manifold with corners is the Lie-algebra $\mathcal{V}_{b}(Z)$ of $b$-vector fields, i.e. smooth vector fields on $Z$ tangent to all boundary hyperfaces $H \in \mathcal{F}_{1}(Z)$ of $Z$. With respect to local coordinates $(x, y): Z \supseteq U \longrightarrow \overline{\mathbb{R}}_{+}^{k} \times \mathbb{R}_{y}^{n-k}$ near a boundary face of codimension $k$, a base of $\left.\mathcal{V}_{b}(Z)\right|_{U}$ over $\mathcal{C}^{\infty}(U)$ is given by the vector fields of the form $x_{j} \partial_{x_{j}}, j=1, \ldots, k$, and $\partial_{y_{\ell}}, \ell=1, \ldots, n-k$. Therefore, there exists a smooth vector bundle ${ }^{b} T Z \longrightarrow Z$ together with a natural map of vector bundles $j^{b}:{ }^{b} T Z \longrightarrow T Z$ such that $\mathcal{V}_{b}(Z)=j^{b}\left(\mathcal{C}^{\infty}\left(Z,{ }^{b} T Z\right)\right)$. The bundle ${ }^{b} T Z$ is called the $b$-tangent bundle. We apply the functor $\Omega^{\alpha}$ of $\alpha$-densities to the b-tangent bundle ${ }^{b} T Z$, and get the bundle ${ }^{b} \Omega^{\alpha}(Z)$ of $b$ - $\alpha$-densities. A choice 
of local coordinates $(x, y)$ as above determines a trivialization of $\left.{ }^{b} \Omega^{\alpha}(Z)\right|_{U}$ by $\left|\frac{d x}{x} d y\right|^{\alpha}=\left|\frac{d x_{1}}{x_{1}} \cdots \frac{d x_{k}}{x_{k}} d y_{1} \cdots d y_{n-k}\right|^{\alpha}$.

The algebra $\operatorname{Diff}_{b}^{*}(Z)$ of $b$-differential operators is defined as the enveloping algebra of the Lie algebra $\mathcal{V}_{b}(Z)$; it is a filtered $\mathcal{C}^{\infty}(Z)$-module. As usual, bdifferential operators acting between sections of vector bundles $E_{1}, E_{2} \longrightarrow Z$ are given by $\operatorname{Diff}_{b}^{*}\left(Z, E_{1}, E_{2}\right):=\operatorname{Diff}_{b}^{*}(Z) \otimes_{\mathcal{C}^{\infty}(Z)} \mathcal{C}^{\infty}\left(Z, \operatorname{Hom}\left(E_{1}, E_{2}\right)\right)$.

For a smooth vector bundle $E \longrightarrow Z$ we denote by $\dot{\mathcal{C}}^{\infty}(Z, E)$ the space of all smooth sections vanishing to infinite order at the boundary faces of $Z$. The space of extendible distributions is defined by $\mathcal{C}^{-\infty}(X, E):=\left(\dot{\mathcal{C}}^{\infty}\left(Z, E^{*} \otimes^{b} \Omega^{1}\right)\right)^{\prime}$.

Let $\varrho_{F}: Z \longrightarrow \overline{\mathbb{R}}_{+}$be a defining function of the boundary face $F \in \mathcal{F}_{1}(Z)$, and $\varrho:=\prod_{F \in \mathcal{F}_{1}(Z)} \varrho_{F}: Z \longrightarrow \overline{\mathbb{R}}_{+}$. Then we call the elements

$$
\mathcal{A}(Z):=\bigcup_{m \in \mathbb{R}}\left\{u \in \mathcal{C}^{-\infty}(Z): \mathcal{V}_{b}(Z)^{k} u \subseteq \varrho^{m} L^{\infty}(Z) \text { for all } k \in \mathbb{N}_{0}\right\}
$$

conormal functions on $Z$. By a form of Sobolev's Lemma, conormal functions are smooth in the interior $Z_{0}:=\operatorname{int} Z$ of $Z$.

2.2. Manifolds with conical singularities. A manifold $M$ with conical singularity $m \in M$ is a topological space such that $\widetilde{M}=M \backslash\{m\}$ is a smooth manifold, and there exists a compact manifold $X$ with boundary together with a homeomorphism $\varphi: M \rightarrow X / \partial X$ which induces a diffeomorphism from $\widetilde{M}$ to the interior $X_{0}$ of $X$. The manifold $X$ is called the stretched manifold associated with $M$. In fact, the analysis of the operators of the cone algebra shall be performed on the stretched manifold $X$. The definition easily extends to manifolds with a finite number of conical points; the associated stretched manifold $X$ is again a compact manifold with boundary. Note that the stretched manifold associated with a manifold with one conical point may coincide with that of another manifold having various singularities. However, the resulting calculi on the stretched manifold are slightly different, the one corresponding to the case of one singularity containing the other.

2.3. The Mellin transform. The Mellin transform is given by

$$
z \mapsto \int_{0}^{\infty} x^{z} u(x) \frac{d x}{x}
$$

for appropriate functions (or distributions) on the real half-axis. Natural domains for the Mellin transform are the spaces

$$
\mathcal{T}_{\gamma}\left(\mathbb{R}_{+}, F\right), \quad \gamma \in \mathbb{R},
$$

consisting of smooth functions $u: \mathbb{R}_{+} \longrightarrow F$ with values in a Fréchet space $F$ such that $x^{\frac{1}{2}-\gamma}|\log x|^{k}||\left(x \partial_{x}\right)^{l} u(x)|| \mid$ is uniformly bounded in $x>0$ for all semi-norms $\|\cdot\| \|$ of $F$ and all $k, l \in \mathbb{N}_{0}$. Then the Mellin transform induces an isomorphism $M_{\gamma}$ of $\mathcal{T}_{\gamma}\left(\mathbb{R}_{+}, F\right)$ to the $F$-valued rapidly decreasing functions on the vertical line $\left\{\operatorname{Re} z=\frac{1}{2}-\gamma\right\}$. 
As an example, if $\omega \in C_{c}^{\infty}\left(\overline{\mathbb{R}}_{+}\right)$equals 1 near $x=0$, then for $\zeta \in \mathbb{C}$ and $k \in \mathbb{N}_{0}$, we have $\omega(x) x^{-\zeta}(\log x)^{k} \in \mathcal{T}_{\gamma}\left(\mathbb{R}_{+}\right)$if and only if $\operatorname{Re} \zeta<\frac{1}{2}-\gamma$. Its Mellin transform

$$
\psi_{\zeta, k}(z)=M_{\gamma}\left(\omega(x) x^{-\zeta}(\log x)^{k}\right)(z)
$$

extends to a meromorphic function with precisely one pole at $\zeta$ of order $k+1$, that decreases rapidly on vertical lines 'outside' the pole. The principal part of the Laurent expansion of $\psi_{\zeta, k}$ at $\zeta$ is given by $(-1)^{k} k !(z-\zeta)^{-(k+1)}$.

2.4. Weighted Sobolev spaces. For a compact manifold $X$ with boundary, we denote by $L^{2}\left(X,{ }^{b} \Omega^{\frac{1}{2}}\right)$ the Hilbert space of all square-integrable b-half densities on $X$, i.e. the completion of $\dot{\mathcal{C}}^{\infty}\left(X,{ }^{b} \Omega^{\frac{1}{2}}\right)$ with respect to the sesquilinear pairing

$$
<f, g>_{L^{2}\left(X,{ }^{b} \Omega^{\frac{1}{2}}\right)}:=\int_{X} f \bar{g} \text { for } f, g \in \dot{\mathcal{C}}^{\infty}\left(X,{ }^{b} \Omega^{\frac{1}{2}}\right) .
$$

Let $\varrho_{H}: X \longrightarrow \overline{\mathbb{R}}_{+}$be a defining function for $H \in \mathcal{F}_{1}(X)$. For a system $\mathfrak{b}_{H} \in \mathbb{R}$, $H \in \mathcal{F}_{1}(X)$, we abbreviate $\varrho^{\mathfrak{b}}:=\prod_{H \in \mathcal{F}_{1}(X)} \varrho_{H}^{\mathfrak{b}}$, and denote by $\varrho^{\mathfrak{b}} L^{2}\left(X,{ }^{b} \Omega^{\frac{1}{2}}\right)$ the Hilbert space of all $f \in \mathcal{C}^{-\infty}\left(X,{ }^{b} \Omega^{\frac{1}{2}}\right)$ with $\varrho^{-b} f \in L^{2}\left(X,{ }^{b} \Omega^{\frac{1}{2}}\right)$. Note that for $\mathfrak{b}_{H} \neq 0$ the Hilbert space structure on $\varrho^{\mathfrak{b}} L^{2}\left(X,{ }^{b} \Omega^{\frac{1}{2}}\right)$ depends on the choice of the defining function $\varrho_{H}$.

Naturally associated to $X$ is the scale of weighted $b$-Sobolev spaces defined for $m \in \mathbb{N}_{0}$ by $[\mathbf{3 1},(5.42),(5.44)]$

$$
\varrho^{\mathfrak{b}} H_{b}^{m}\left(X,{ }^{b} \Omega^{\frac{1}{2}}\right):=\left\{f \in \varrho^{\mathfrak{b}} L^{2}\left(X,{ }^{b} \Omega^{\frac{1}{2}}\right): \operatorname{Diff}_{b}^{m}\left(X,{ }^{b} \Omega^{\frac{1}{2}}\right) f \subseteq \varrho^{\mathfrak{b}} L^{2}\left(X,{ }^{b} \Omega^{\frac{1}{2}}\right)\right\} .
$$

Here $a \in \operatorname{Diff}_{b}^{m}\left(X,{ }^{b} \Omega^{\frac{1}{2}}\right)$ acts on $f \in \varrho^{\mathfrak{b}} L^{2}\left(X,{ }^{b} \Omega^{\frac{1}{2}}\right) \subseteq \mathcal{C}^{-\infty}\left(X,{ }^{b} \Omega^{\frac{1}{2}}\right)$ in the distributional sense. Since $\operatorname{Diff}_{b}^{m}$ is a finitely generated $\overline{\mathcal{C}}^{\infty}(X)$-module, the spaces $\varrho^{\mathfrak{b}} H_{b}^{m}\left(X,{ }^{b} \Omega^{\frac{1}{2}}\right)$ are in a non-natural way Hilbert spaces - see $[\mathbf{1 4}],[\mathbf{1 8}]$ for more details.

For $\gamma \in \mathbb{R}$ it is useful to consider also the spaces

$$
\mathcal{H}^{m, \gamma}\left(X,{ }^{b} \Omega^{\frac{1}{2}}\right):=\varrho^{\mathfrak{b}_{\gamma}} H_{b}^{m}\left(X,{ }^{b} \Omega^{\frac{1}{2}}\right)
$$

with $\left(\mathfrak{b}_{\gamma}\right)_{H}=\gamma-\frac{n}{2}$ for all $H \in \mathcal{F}_{1}(X)$. By [18, Proposition 2.2.2] and [57, Theorem 1.1.21] this definition coincides with that given in [57, Definition 1.1.19]. Note that the scale of Sobolev spaces $\varrho^{\mathfrak{b}} H_{b}^{m}\left(X,{ }^{b} \Omega^{\frac{1}{2}}\right)$ and $\mathcal{H}^{m, \gamma}\left(X,{ }^{b} \Omega^{\frac{1}{2}}\right)$ can be extended to all $m \in \mathbb{R}$ either by duality and interpolation or by defining [41]

$$
\varrho^{\mathfrak{b}} H_{b}^{m}\left(X,{ }^{b} \Omega^{\frac{1}{2}}\right):=\left\{f \in \mathcal{C}^{-\infty}\left(X,{ }^{b} \Omega^{\frac{1}{2}}\right): \Psi_{b, c l}^{m}\left(X,{ }^{b} \Omega^{\frac{1}{2}}\right) f \subseteq \varrho^{\mathfrak{b}} L^{2}\left(X,{ }^{b} \Omega^{\frac{1}{2}}\right)\right\},
$$

where $\Psi_{b, c l}^{m}\left(X,{ }^{b} \Omega^{\frac{1}{2}}\right)$ is the space of classical b-pseudodifferential operators defined in Definition 4.7. Also it is possible to give a definition in terms of local coordinates, cf. [4, Section 7.1.2]. Note that each scalar product in $\mathcal{H}^{0,0}\left(X,{ }^{b} \Omega^{\frac{1}{2}}\right)$ induces a nondegenerate sesqui-linear pairing

$$
\langle\cdot, \cdot\rangle_{0,0}: \mathcal{H}^{s, \gamma}\left(X, \Omega^{\frac{1}{2}}\right) \times \mathcal{H}^{-s,-\gamma}\left(X, \Omega^{\frac{1}{2}}\right) \rightarrow \mathbb{C}
$$




\section{Review on the cone algebra with discrete asymptotics}

The cone algebra consists of pseudodifferential operators in the interior of $M$ that have a specific behavior near the singularity. Passing to the streched manifold $X$ with a splitting of coordinates near the boundary, the cone operators are modulo certain smoothing remainders - Mellin pseudodifferential operators build upon symbols that extend in the covariable meromorphically to the whole complex plane. Note that this description does not depend on the choice of the coordinates as long as the coordinate change extends to a diffeomorphism of $X$, cf. [12] and also [46]. For simplicity we restrict ourselves to the case of $M$ having only one conical singularity; this situation includes all basic ideas and the general case of finitely many singularities is a straightforward extension. Note that the case of non-connected boundary $\partial X$ is not excluded.

Most of the here presented material can be found, for example, in the monographs [57] and [58], though our presentation uses slightly different notation. Moreover, to formulate the connection of Melrose's and Schulze's approach in a smooth way, we introduce a 'weak' type of the cone algebra.

3.1. Spaces with asymptotics and meromorphic Mellin symbols. In this subsection let $Y$ be a closed manifold (later, $Y$ plays the role of the boundary of $X)$. Furthermore, let $\Omega^{\frac{1}{2}}=\Omega^{\frac{1}{2}}(Y)$ be the complex bundle of $\frac{1}{2}$-densities over $Y$, and $\Psi_{c l}^{m}\left(Y, \Omega^{\frac{1}{2}}\right)$ be the Fréchet space of classical (or polyhomogeneous) pseudodifferential operators of order $m$, acting in $\mathcal{C}^{\infty}\left(Y, \Omega^{\frac{1}{2}}\right)$, the smooth sections of $\Omega^{\frac{1}{2}} \cdot \Psi_{c l}^{m}\left(Y, \Omega^{\frac{1}{2}} ; \mathbb{R}\right)$ is the space of parameter-dependent operators; here, the real parameter enters (in the local symbol estimates) as an additional covariable.

Note that if $Y=\biguplus_{j=1}^{L} Y_{j}$ consists of several connected components, each pseudodifferential operator $b \in \Psi_{c l}^{m}\left(Y, \Omega^{\frac{1}{2}}\right)$ can be identified - via localizations to the different components - with a square matrix $\left(b_{j k}\right)_{j, k=1, \ldots, L}$ of operators such hat $b_{j j} \in \Psi_{c l}^{m}\left(Y_{j}, \Omega^{\frac{1}{2}}\right)$ and the off-diagonal elements $b_{j k}$ have kernels in the space $\mathcal{C}^{\infty}\left(Y_{j} \times Y_{k}, \pi_{1}^{*} \Omega^{\frac{1}{2}} \otimes \pi_{2}^{*} \Omega^{\frac{1}{2}}\right)$, where $\pi_{1}$ and $\pi_{2}$ are the projections of $Y_{j} \times Y_{k}$ to the first and second component, respectively. Similar facts hold for the parameterdependent class. The off-diagonal elements then depend rapidly decreasing on the parameter.

Definition 3.1. Let $\pi_{\mathbb{C}}: \mathbb{C} \times \rightarrow \mathbb{C}$ be the projection to the first component. A discrete set $P \subset \mathbb{C} \times \mathbb{N}$ is called a weak asymptotic type for Mellin symbols if $P=\left\{\left(p, n_{p}\right): p \in \pi_{\mathbb{C}} P\right\}$ and $\left|\operatorname{Re} p_{j}\right| \rightarrow \infty$ whenever $\left(p_{j}\right)_{j \in \mathbb{N}}$ is a sequence in $\pi_{\mathbb{C}} P$ with $\left|p_{j}\right| \rightarrow \infty$. With such a $P$ we associate the space $M_{P, w}^{m}\left(Y, \Omega^{\frac{1}{2}}\right)$ of all meromorphic functions $h: \mathbb{C} \backslash \pi_{\mathbb{C}} P \rightarrow \Psi_{c l}^{m}\left(Y, \Omega^{\frac{1}{2}}\right)$ such that for each $N \in \mathbb{N}$ and 
appropriate elements $r_{p, k} \in \Psi^{-\infty}\left(Y, \Omega^{\frac{1}{2}}\right)$

$$
h_{N}(\beta, \tau):=h(\beta+i \tau)-\sum_{p \in \pi_{\mathbb{C}} P,|\operatorname{Re} p| \leq N} \sum_{k=0}^{n_{p}} r_{p, k} \psi_{p, k}(\beta+i \tau)
$$

is a continuous function of $\beta \in[-N, N]$ with values in $\Psi_{c l}^{m}\left(Y, \Omega^{\frac{1}{2}} ; \mathbb{R}_{\tau}\right)$. Here the $\psi_{p, k}$ are as in (2.4). If, additionally, for each $p \in \pi_{\mathbb{C}} P, N_{p} \subset \Psi^{-\infty}\left(Y, \Omega^{\frac{1}{2}}\right)$ is a finite-dimensional subspace of finite rank operators, we say that the set

$$
P=\left\{\left(p, n_{p}, N_{p}\right): p \in \pi_{\mathbb{C}} P\right\}
$$

is an asymptotic type for Mellin symbols, and we denote by $M_{P}^{m}\left(Y, \Omega^{\frac{1}{2}}\right)$ the subspace of all $h \in M_{P, w}^{m}\left(Y, \Omega^{\frac{1}{2}}\right)$ with $r_{p, k} \in N_{p}$. The unique asymptotic type associated to $\pi_{\mathbb{C}} P=\emptyset$ is denoted by $O$.

For brevity, we restrict ourselves mostly to the case of asymptotic types; the obvious modifications for weak asymptotic types are left to the reader. The projective limit topology under the maps $h \mapsto h_{N}$ and $h \mapsto r_{p, k}(h)$ turns $M_{P}^{m}\left(Y, \Omega^{\frac{1}{2}}\right)$ into a Fréchet space.

According to the above mentioned representation of pseudodifferential operators in the case $Y=\biguplus_{j=1}^{P} Y_{j}$, functions of $M_{P}^{m}\left(Y, \Omega^{\frac{1}{2}}\right)$ have a corresponding representation as block-matrices of functions. The off-diagonal terms then in fact have values in the smoothing operators. Therefore, the matrices with entries only in the $(j, k)$-th position $(j \neq k)$ form a subspace of $M_{P}^{-\infty}\left(Y, \Omega^{\frac{1}{2}}\right)$, denoted by

$$
M_{P}^{-\infty}\left(Y_{j}, Y_{k}, \Omega^{\frac{1}{2}}\right) \text {. }
$$

3.2. Mellin pseudodifferential operators. Let $Y$ be a compact manifold and $\Gamma_{\nu}=\{z: \operatorname{Re} z=\nu\}$. If $h \in \mathcal{C}^{\infty}\left(\mathbb{R}_{+}, L^{m}\left(Y ; \Gamma_{\frac{1}{2}-\gamma}\right)\right)$, where we identify the parameter space $\Gamma_{\frac{1}{2}-\gamma}$ with $\mathbb{R}$, and $\left\|\left(x \partial_{x}\right)^{k} h(x)\right\|||$ is uniformly bounded in $x>0$ for each continuous semi-norm of $\Psi^{m}\left(Y ; \Gamma_{\frac{1}{2}-\gamma}\right)$ and all $k \in \mathbb{N}_{0}$, then

$$
\begin{aligned}
{\left[\operatorname{op}_{M}^{(\gamma)}(h) u\right](x) } & =\frac{1}{2 \pi i} \int_{\Gamma_{\frac{1}{2}-\gamma}} x^{-z} h(x, z)\left(M_{\gamma} u\right)(z) d z \\
& =\int_{\mathbb{R}_{\xi}} \int_{0}^{\infty}\left(\frac{x^{\prime}}{x}\right)^{\frac{1}{2}-\gamma+i \xi} h\left(x, \frac{1}{2}-\gamma+i \xi\right) u\left(x^{\prime}\right) \frac{d x^{\prime}}{x^{\prime}} d \xi
\end{aligned}
$$

with convergence of the integral in $\mathcal{C}^{\infty}\left(Y, \Omega^{\frac{1}{2}}\right)$, defines a continuous operator

$$
\operatorname{op}_{M}^{(\gamma)}(h): \mathcal{T}_{\gamma}\left(\mathbb{R}_{+}, \mathcal{C}^{\infty}\left(Y, \Omega^{\frac{1}{2}}\right)\right) \rightarrow \mathcal{T}_{\gamma}\left(\mathbb{R}_{+}, \mathcal{C}^{\infty}\left(Y, \Omega^{\frac{1}{2}}\right)\right) .
$$

In particular, we may take $h$ from the space $\mathcal{C}_{c}^{\infty}\left(\overline{\mathbb{R}}_{+}, M_{P, w}^{m}\left(Y, \Omega^{\frac{1}{2}}\right)\right)$, cf. Definition 3.1 , provided $\pi_{\mathbb{C}} P \cap \Gamma_{\frac{1}{2}-\gamma}=\emptyset$. 


\subsection{Test functions and residual Green operators.}

Definition 3.2. For $\gamma \in \mathbb{R}$, let us introduce the Fréchet space

$$
\mathcal{C}_{\gamma}^{\infty}\left(X,{ }^{b} \Omega^{\frac{1}{2}}\right)=\bigcap_{k, m \in \mathbb{N}_{0}}(\log \varrho)^{-k} \mathcal{H}^{m, \gamma}\left(X,{ }^{b} \Omega^{\frac{1}{2}}\right),
$$

cf. Section 2.4. Because of $\left[\log \varrho, \operatorname{Diff}_{b}^{m}\left(X,{ }^{b} \Omega^{\frac{1}{2}}\right)\right] \subseteq \operatorname{Diff}_{b}^{m-1}\left(X,{ }^{b} \Omega^{\frac{1}{2}}\right)$, this space coincides with

$$
\left\{f \in \varrho^{\mathfrak{b}_{\gamma}} L^{2}\left(X,{ }^{b} \Omega^{\frac{1}{2}}\right): \operatorname{Diff}_{b}^{m}\left(X,{ }^{b} \Omega^{\frac{1}{2}}\right) f \subseteq(\log \varrho)^{-k} \varrho^{\mathfrak{b}_{\gamma}} L^{2}\left(X,{ }^{b} \Omega^{\frac{1}{2}}\right) \forall k, m \in \mathbb{N}_{0}\right\}
$$

and does not depend on the choice of the boundary defining function.

The index $\gamma$ encodes the flatness of sections at the boundary in the following sense $\mathcal{C}_{\gamma}^{\infty}\left(X,{ }^{b} \Omega^{\frac{1}{2}}\right)=\varrho^{\gamma} \mathcal{C}_{0}^{\infty}\left(X,{ }^{b} \Omega^{\frac{1}{2}}\right)$. In particular,

$$
\dot{\mathcal{C}}^{\infty}\left(X,{ }^{b} \Omega^{\frac{1}{2}}\right)=\bigcap_{\gamma \in \mathbb{R}} \mathcal{C}_{\gamma}^{\infty}\left(X,{ }^{b} \Omega^{\frac{1}{2}}\right)
$$

These spaces will serve as natural domains the cone operators act on. Moreover, we use them to describe a certain residual class of smoothing cone operators. To this end, fix a boundary defining function $\varrho$, let $U$ be a collar neighborhood of $\partial X$ and $(x, y): U \stackrel{\cong}{\longrightarrow} \overline{\mathbb{R}}_{+} \times \partial X$ be coordinates with $\varrho(x, y)=x$. These coordinates give an identification of sections of $\mathcal{C}_{\gamma}^{\infty}\left(X,{ }^{b} \Omega^{\frac{1}{2}}\right)$ supported near the boundary and functions with support bounded from above in $\mathcal{T}_{\gamma-\frac{n-1}{2}}\left(\mathbb{R}_{+}, \mathcal{C}^{\infty}\left(\partial, \Omega^{\frac{1}{2}}\right)\right)$, cf. (2.3).

Definition 3.3. Let $\gamma \in \mathbb{R}, k \in \mathbb{N}$, and $\pi_{\mathbb{C}}: \mathbb{C} \times \rightarrow \mathbb{C}$ be the projection on the first component. A finite set $Q=\left\{\left(q, l_{p}\right): q \in \pi_{\mathbb{C}} Q\right\} \subset \mathbb{C} \times \mathbb{N}_{0}$ is called a weak asymptotic type with respect to $(\gamma, k)$ if $\pi_{\mathbb{C}} Q \subset\left\{\frac{n}{2}-\gamma-k<\operatorname{Re}(q)<\frac{n}{2}-\gamma\right\}$, and with $(q, l) \in Q$ also $(q-1, l) \in Q$ provided Re $q-1>\frac{n}{2}-\gamma-k$ (the latter means that $Q$ satisfies the so-called shadow condition). The collection of all such sets is denoted by $\operatorname{As}_{w}(\gamma, k)$. We write $O$ (or sometimes $O_{k}$ ) for the unique asymptotic type corresponding to $\pi_{\mathbb{C}} Q=\emptyset$ with respect to the weight data $(\gamma, k)$.

Definition 3.4. For $Q=\left\{\left(q, l_{q}\right): q \in \pi_{\mathbb{C}} Q\right\} \in \operatorname{As}_{w}(\gamma, k)$, let $\mathcal{C}_{\gamma, Q, w}^{\infty}\left(X,{ }^{b} \Omega^{\frac{1}{2}}\right)$ be the Fréchet subspace of $\mathcal{C}_{\gamma}^{\infty}\left(X,{ }^{b} \Omega^{\frac{1}{2}}\right)$ consisting of all $f$ such that

$$
f-\omega(x) \sum_{q \in \pi_{\mathbb{C}} Q} \sum_{l=0}^{l_{q}} f_{q, l} x^{-q}(\log x)^{l} \in \mathcal{C}_{\gamma+k-\varepsilon}^{\infty}\left(X,{ }^{b} \Omega^{\frac{1}{2}}\right) \quad \forall \varepsilon>0
$$

with coefficients $f_{q, l} \in \mathcal{C}^{\infty}\left(\partial X, \Omega^{\frac{1}{2}}\right)$, and $\omega \in \mathcal{C}_{c}^{\infty}\left(\overline{\mathbb{R}}_{+}\right)$with $\omega \equiv 1$ near $x=0$.

If, additionally, for each $q \in \pi_{\mathbb{C}} Q, L_{q} \subseteq \mathcal{C}^{\infty}\left(\partial X, \Omega^{\frac{1}{2}}\right)$ is a finite-dimensional subspace, we call

$$
Q=\left\{\left(q, l_{q}, L_{q}\right): q \in \pi_{\mathbb{C}} Q\right\}
$$

an asymptotic type with respect to $(\gamma, k)$ and we denote by $\mathcal{C}_{\gamma, Q}^{\infty}\left(X,{ }^{b} \Omega^{\frac{1}{2}}\right)$ the subspace of all $f \in \mathcal{C}_{\gamma, Q, w}^{\infty}\left(X,{ }^{b} \Omega^{\frac{1}{2}}\right)$ with $f_{q, l} \in L_{q}$. The set of all asymptotic types is denoted by $\operatorname{As}(\gamma, k)$. 
In analogy, we can define Sobolev spaces with asymptotics $\mathcal{H}_{Q}^{s, \gamma}\left(X, \Omega^{\frac{1}{2}}\right)$ and $\mathcal{H}_{Q, w}^{s, \gamma}\left(X, \Omega^{\frac{1}{2}}\right)$, which are Fréchet subspaces of $\mathcal{H}^{s, \gamma}\left(X, \Omega^{\frac{1}{2}}\right)$, by requiring the difference in (3.3) to belong to $\mathcal{H}^{s, \gamma+k-\varepsilon}\left(X, \Omega^{\frac{1}{2}}\right)$ for each positive $\varepsilon$.

Similar notations make sense for the case $k=\infty$. Then $Q$ is a (weak) asymptotic type with respect to $(\gamma, \infty)$ if each $Q_{k}$ defined by

$$
\pi_{\mathbb{C}} Q_{k}=\pi_{\mathbb{C}} Q \cap\left\{\frac{n}{2}-\gamma-k<\operatorname{Re}(q)<\frac{n}{2}-\gamma\right\}
$$

is a (weak) asymptotic type for $(\gamma, k)$, and the corresponding spaces are introduced as the projective limit of the spaces with respect to $Q_{k}$, for example,

$$
\mathcal{C}_{\gamma, Q}^{\infty}\left(X,{ }^{b} \Omega^{\frac{1}{2}}\right)=\bigcap_{k \in \mathbb{N}} \mathcal{C}_{\gamma, Q_{k}}^{\infty}\left(X,{ }^{b} \Omega^{\frac{1}{2}}\right) .
$$

Remark 3.5. Note that the space $\mathcal{C}_{\gamma, Q, w}^{\infty}\left(X,{ }^{b} \Omega^{\frac{1}{2}}\right)$ does not depend on the choice of coordinates near the boundary, whereas $\mathcal{C}_{\gamma, Q}^{\infty}\left(X,{ }^{b} \Omega^{\frac{1}{2}}\right)$ does. However, passing to another boundary defining function $\varrho^{\prime}$ and corresponding coordinates, $\mathcal{C}_{\gamma, Q}^{\infty}\left(X,{ }^{b} \Omega^{\frac{1}{2}}\right)$ transforms to $\mathcal{C}_{\gamma, Q^{\prime}}^{\infty}\left(X,{ }^{b} \Omega^{\frac{1}{2}}\right)$ with an asymptotic type $Q^{\prime} \in \operatorname{As}(\gamma, k)$, which can be calculated explicitly from $Q$, cf. for example [48], Remark 3.2.2. The same is true for Sobolev spaces with asymptotics.

Definition 3.6. Let $\gamma, \gamma^{\prime} \in \mathbb{R}$ and $k \in \mathbb{N} \cup\{\infty\}$. If $Q \in \operatorname{As}(-\gamma, k)$ and $Q^{\prime} \in \operatorname{As}\left(\gamma^{\prime}, k\right)$ are asymtotic types, then $\mathfrak{C}_{G}\left(X,{ }^{b} \Omega^{\frac{1}{2}},\left(\gamma, \gamma^{\prime}, k\right)\right)_{Q^{\prime}, Q}$ consists of all operators $G \in \cap_{s \in \mathbb{R}} \mathcal{L}\left(\mathcal{H}^{s, \gamma}\left(X,{ }^{b} \Omega^{\frac{1}{2}}\right), \mathcal{H}^{s, \gamma^{\prime}}\left(X,{ }^{b} \Omega^{\frac{1}{2}}\right)\right)$ such that

$$
G: \mathcal{H}^{s, \gamma}\left(X,{ }^{b} \Omega^{\frac{1}{2}}\right) \rightarrow \mathcal{C}_{\gamma^{\prime}, Q^{\prime}}\left(X,{ }^{b} \Omega^{\frac{1}{2}}\right), \quad G^{*}: \mathcal{H}^{s,-\gamma^{\prime}}\left(X,{ }^{b} \Omega^{\frac{1}{2}}\right) \rightarrow \mathcal{C}_{-\gamma, Q}^{\infty}\left(X,{ }^{b} \Omega^{\frac{1}{2}}\right)
$$

for all real $s$. Here, ${ }^{*}$ denotes the adjoint in $\mathcal{H}^{0,0}\left(X,{ }^{b} \Omega^{\frac{1}{2}}\right)=\varrho^{b_{0}} L^{2}\left(X,{ }^{b} \Omega^{\frac{1}{2}}\right)$. The corresponding weak version is denoted by the additional subscript $w$. The uniuon over all types $Q, Q^{\prime}$ is denoted by $\mathfrak{C}_{G}\left(X,{ }^{b} \Omega^{\frac{1}{2}},\left(\gamma, \gamma^{\prime}, k\right)\right)$. Such operators will be refered to as (weak) Green operators.

There is another possibility to characterize Green operators, namely as integral operators with respect to the $\mathcal{H}^{0,0}\left(X,{ }^{b} \Omega^{\frac{1}{2}}\right)$-scalar product with kernels in certain spaces with asymptotics. To be precise, if $f \otimes g$ is a pure tensor with $f \in \mathcal{C}_{\gamma^{\prime}, Q^{\prime}}^{\infty}\left(X,{ }^{b} \Omega^{\frac{1}{2}}\right)$ and $g \in \mathcal{C}_{-\gamma, Q}^{\infty}\left(X,{ }^{b} \Omega^{\frac{1}{2}}\right)$ we can consider the continuous operator $u \mapsto\langle g, \bar{u}\rangle_{0,0} f: \mathcal{H}^{s, \gamma}\left(X,{ }^{b} \Omega^{\frac{1}{2}}\right) \rightarrow \mathcal{H}^{s, \gamma^{\prime}}\left(X,{ }^{b} \Omega^{\frac{1}{2}}\right)$. This operation can be extended to the completed $\pi$-tensor product. Now it can be shown, cf. [59], [64], that $G$ is as in Definition 3.6 if and only if it has a kernel

$$
k_{G} \in \mathcal{C}_{\gamma^{\prime}, Q^{\prime}}^{\infty}\left(X,{ }^{b} \Omega^{\frac{1}{2}}\right) \widehat{\otimes}_{\pi} \mathcal{C}_{-\gamma, \bar{Q}}^{\infty}\left(X,{ }^{b} \Omega^{\frac{1}{2}}\right),
$$

where $\bar{Q}$ is the complex conjugate asymptotic type to $Q$, i.e. $\bar{Q}=\{(\bar{q}, l, \bar{L})\}$, provided $Q=\{(q, l, L)\}$.

The analogous characterization holds for weak Green operators as we shall show in Subsection 5.3 below. 
3.4. The cone algebra with discrete asymptotics. Recall that we have fixed a splitting of coordinates $(x, y)$ near the boundary of $X$, and identified sections $X \rightarrow{ }^{b} \Omega^{\frac{1}{2}}$ near the boundary with functions on $\mathbb{R}+$ taking values in sections $\partial X \rightarrow \Omega^{\frac{1}{2}}$, cf. Subsection 3.3. This identification will be suppressed from the notation. Also recall that $n$ denotes the dimension of $X$.

Let us first introduce the residual cone pseudodifferential operators.

Definition 3.7. Let $\gamma, m \in \mathbb{R}, j \in \mathbb{N}_{0}, k \in \mathbb{N}$. Then $\mathfrak{C}_{M+G}^{m-j}\left(X,{ }^{b} \Omega^{\frac{1}{2}},(\gamma, \gamma-\right.$ $m, k)$ ) consists of all operators $M+G$ with $G \in \mathfrak{C}_{G}\left(X,{ }^{b} \Omega^{\frac{1}{2}},(\gamma, \gamma-m, k)\right)$ and a smoothing Mellin operator

$$
M=\omega_{0}\left\{\sum_{l=0}^{k-j-1} x^{-m+j+l} \operatorname{op}_{M}^{\left(\gamma_{l}-\frac{n-1}{2}\right)}\left(h_{l}\right)\right\} \omega_{1},
$$

where $h_{l} \in M_{P_{l}}^{-\infty}\left(\partial X, \Omega^{\frac{1}{2}}\right), \gamma-j-l \leq \gamma_{l} \leq \gamma$, and the asymptotic types $P_{l}$ satisfy $\pi_{\mathbb{C}} P_{l} \cap \Gamma_{\frac{n}{2}-\gamma_{l}}=\emptyset$. Moreover, $\omega_{0}, \omega_{1} \in \mathcal{C}_{c}^{\infty}\left(\overline{\mathbb{R}}_{+}\right)$are identically 1 near $x=0$. For the case $k=\infty$ we set

$$
\mathfrak{C}_{M+G}^{m-j}\left(X,{ }^{b} \Omega^{\frac{1}{2}},(\gamma, \gamma-m, \infty)\right)=\bigcap_{k \in \mathbb{N}} \mathfrak{C}_{M+G}^{m-j}\left(X,{ }^{b} \Omega^{\frac{1}{2}},(\gamma, \gamma-m, k)\right) .
$$

Again the corresponding weak versions are signed by the additional subscript $w$.

Definition 3.8. Let $\gamma, m \in \mathbb{R}, j \in \mathbb{N}_{0}$, and $k \in \mathbb{N} \cup\{\infty\}$. The 'cone algebra' $\mathfrak{C}^{m-j}\left(X,{ }^{b} \Omega^{\frac{1}{2}},(\gamma, \gamma-m, k)\right)$ of order $m-j$ with respect to the data $(\gamma, \gamma-m, k)$ consists of all continuous operators $a: \mathcal{C}_{\gamma}^{\infty}\left(X,{ }^{b} \Omega^{\frac{1}{2}}\right) \rightarrow \mathcal{C}_{\gamma-m}^{\infty}\left(X,{ }^{b} \Omega^{\frac{1}{2}}\right)$ of the form

$$
a=\omega_{0} x^{-m+j} \operatorname{op}_{M}^{\left(\gamma-\frac{n-1}{2}\right)}(h) \omega_{1}+\left(1-\omega_{2}\right) a_{\psi}\left(1-\omega_{3}\right)+M+G,
$$

with a holomorphic Mellin symbol $h \in \mathcal{C}^{\infty}\left(\overline{\mathbb{R}}_{+}, M_{O}^{m-j}\left(\partial X, \Omega^{\frac{1}{2}}\right)\right)$, a pseudodifferential operator $a_{\psi} \in \Psi_{c l}^{m-j}\left(X_{0},{ }^{b} \Omega^{\frac{1}{2}}\right)$ on the interior $X_{0}$ of $X$, and $M+G \in$ $\mathfrak{C}_{M+G}\left(X,{ }^{b} \Omega^{\frac{1}{2}},(\gamma, \gamma-m, k)\right)$. Moreover, the $\omega_{k}$ 's are arbitrary functions in $\mathcal{C}_{c}^{\infty}\left(\overline{\mathbb{R}}_{+}\right)$ that are identically 1 near $x=0$. The corresponding weak versions of the spaces above are defined accordingly, and marked by an additional index $w$.

Definition 3.9. For $k \in \mathbb{N} \cup\{\infty\}$, we introduce the holomorphic "cone algebra' $\mathfrak{C}^{m-j}\left(X,{ }^{b} \Omega^{\frac{1}{2}},(\gamma, \gamma-m, k)\right)_{O}$ as the space of all $a \in \mathbb{C}^{m-j}\left(X,{ }^{b} \Omega^{\frac{1}{2}},(\gamma, \gamma-m, k)\right)$ of the form

$$
a=\omega_{0} x^{-m+j} \operatorname{op}_{M}^{\left(\gamma-\frac{n-1}{2}\right)}(h) \omega_{1}+(1-\omega) a_{\psi}\left(1-\omega_{2}\right)+G,
$$

with Green operators $G \in \mathfrak{C}_{G}\left(X,{ }^{b} \Omega^{\frac{1}{2}},(\gamma, \gamma-m, k)\right)_{O, O}$.

Note that, since no non-trivial asymptotic respectively meromorphic structure is involved, there is no weak version of the holomorphic algebra.

Using the kernel characterisation of Subsection 3.3 and the identity (3.2), we see that the Green operators in the class $\mathfrak{C}^{m-j}\left(X,{ }^{b} \Omega^{\frac{1}{2}},(\gamma, \gamma-m, \infty)\right)_{O}$ are precisely the integral operators with kernel in $\dot{\mathcal{C}}^{\infty}\left(X^{2},{ }^{b} \Omega^{\frac{1}{2}}\right)$. Furthermore, by Cauchy's integral formula, the operator op ${ }_{M}^{(\gamma)}(h): \dot{\mathcal{C}}^{\infty}\left(X,{ }^{b} \Omega^{\frac{1}{2}}\right) \rightarrow \dot{\mathcal{C}}^{\infty}\left(X,{ }^{b} \Omega^{\frac{1}{2}}\right)$ 
is independent of $\gamma \in \mathbb{R}$, provided $h \in \mathcal{C}^{\infty}\left(\overline{\mathbb{R}}_{+}, M_{O}^{m}\left(\partial X, \Omega^{\frac{1}{2}}\right)\right)$ is a holomorphic Mellin symbol. Hence, considered as operators in $\dot{\mathcal{C}}^{\infty}\left(X,{ }^{b} \Omega^{\frac{1}{2}}\right)$, the cone algebra $\mathfrak{C}^{m-j}\left(X,{ }^{b} \Omega^{\frac{1}{2}},(\gamma, \gamma-m, \infty)\right)_{O}$ does only depend on $m-j$ and not on $(\gamma, \gamma-m)$.

3.5. Symbolic structure and basic properties of the cone algebra. With each operator $a \in \mathfrak{C}^{m}\left(X,{ }^{b} \Omega^{\frac{1}{2}},(\gamma, \gamma-m, k)\right)$ we associate two (principal) symbols. The first one is the conormal symbol, defined by

$$
\begin{aligned}
\sigma_{M}^{(m)}: \mathfrak{C}^{m}\left(X,{ }^{b} \Omega^{\frac{1}{2}},\left(\gamma, \gamma-m, \Theta_{k}\right)\right) & \longrightarrow \Psi^{m}\left(\partial X, \Omega^{\frac{1}{2}} ; \Gamma_{\frac{n}{2}-\gamma}\right): \\
a & \left.\longmapsto\left(h(0)+h_{0}\right)\right|_{\Gamma_{\frac{n}{2}-\gamma}},
\end{aligned}
$$

where $a$ is as in Definition 3.8. Since, in particular, the cone algebra is a subclass of the classical pseudodifferential operators on the interior of $X$ we an associate with each cone operator $a$ the homogeneous principal symbol $\sigma_{\psi}^{(m)}\left(\varrho^{m} a\right)$ defined on $T^{*} X_{0} \backslash X_{0}$. If $\iota:\left.{ }^{b} T^{*} X\right|_{X_{0}} \rightarrow T^{*} X_{0}$ denotes the inverse of the mapping adjoint to $j^{b}:{ }^{b} T X \rightarrow T X$, cf. Subsection 2.1, $\sigma_{\psi}^{(m)}\left(\varrho^{m} a\right) \circ \iota:\left.{ }^{b} T^{*} X\right|_{X_{0}} \backslash\{0\} \rightarrow \mathbb{C}$ extends to a smooth, positively homogeneous map $\sigma_{\psi \cdot \mathbb{C}}^{(m)}(a):{ }^{b} T^{*} X \backslash\{0\} \rightarrow \mathbb{C}$. In other words, in the coordinates $(x, y)$ near the boundary, the homogeneous principal symbol of $a$ has the form

$$
\sigma_{\psi}^{(m)}(a)(x, y, \tau, \eta)=x^{-m} p(x, y, x \tau, \eta)
$$

with $p$ smooth up to $x=0$ and positively homogeneous of degree $m$ in $(\tau, \eta)$. Since positively homogeneous maps on ${ }^{b} T^{*} X \backslash\{0\}$ are determined by their values on the corresponding sphere bundle ${ }^{b} S^{*} X$, we thus obtain the homogeneous principal symbol map

$$
\mathfrak{C}^{m}\left(X,{ }^{b} \Omega^{\frac{1}{2}},\left(\gamma, \gamma-m, \Theta_{k}\right)\right) \rightarrow \mathcal{C}^{\infty}\left({ }^{b} S^{*} X\right): a \mapsto \sigma_{\psi, \mathfrak{C}}^{(m)}(a) .
$$

Let us now summarize the basic properties of the cone algebra. The composition of cone operators yields mappings

$$
\begin{gathered}
\mathfrak{C}^{m^{\prime}-j^{\prime}}\left(X,{ }^{b} \Omega^{\frac{1}{2}},\left(\gamma-m, \gamma-m-m^{\prime}, k\right)\right) \times \mathfrak{C}^{m-j}\left(X,{ }^{b} \Omega^{\frac{1}{2}},(\gamma, \gamma-m, k)\right) \rightarrow \\
\mathfrak{C}^{m+m^{\prime}-\left(j+j^{\prime}\right)}\left(X,{ }^{b} \Omega^{\frac{1}{2}},\left(\gamma, \gamma-\left(m-m^{\prime}\right), k\right)\right),
\end{gathered}
$$

and taking the adjoint with respect to the scalar product in $\mathcal{H}^{0,0}\left(X,{ }^{b} \Omega^{\frac{1}{2}}\right)$ yields mappings

$$
\mathfrak{C}^{m-j}\left(X,{ }^{b} \Omega^{\frac{1}{2}},(\gamma, \gamma-m, k)\right) \rightarrow \mathfrak{C}^{m-j}\left(X,{ }^{b} \Omega^{\frac{1}{2}},(-\gamma+m,-\gamma, k)\right) .
$$

Analogous statements hold for the weak and holomorphic classes.

Each element $a \in \mathbb{C}^{m}\left(X,{ }^{b} \Omega^{\frac{1}{2}},(\gamma, \gamma-m, k)\right)$ induces for each $s \in \mathbb{R}$ and each $Q \in \operatorname{As}(\gamma, k)$ continuous operators

$$
\mathcal{H}^{s, \gamma}\left(X,{ }^{b} \Omega^{\frac{1}{2}}\right) \rightarrow \mathcal{H}^{s-m, \gamma-m}\left(X,{ }^{b} \Omega^{\frac{1}{2}}\right), \quad \mathcal{H}_{Q}^{s, \gamma}\left(X,{ }^{b} \Omega^{\frac{1}{2}}\right) \rightarrow \mathcal{H}_{Q^{\prime}}^{s-m, \gamma-m}\left(X,{ }^{b} \Omega^{\frac{1}{2}}\right),
$$

with a resulting asymptotic type $Q^{\prime} \in \operatorname{As}(\gamma-m, k)$ depending on $Q$ and $a$. These operators are Fredholm if and only if the conormal and homogeneous principal 
symbol of $a$ are invertible. In this case $a$ has a parametrix modulo Green operators. Consequently, we have elliptic regularity in the cone Sobolev spaces (with asymptotics), i.e. if $a u=f$ with an elliptic $a$ and $f \in \mathcal{H}^{s-m, \gamma-m}\left(X,{ }^{b} \Omega^{\frac{1}{2}}\right)(f \in$ $\left.\mathcal{H}_{Q^{\prime}}^{s-m, \gamma-m}\left(X,{ }^{b} \Omega^{\frac{1}{2}}\right)\right)$ and $u$ in some $\mathcal{H}^{r, \gamma}\left(X,{ }^{b} \Omega^{\frac{1}{2}}\right)$, then actually $u \in \mathcal{H}^{s, \gamma}\left(X,{ }^{b} \Omega^{\frac{1}{2}}\right)$ $\left(u \in \mathcal{H}_{Q}^{s, \gamma}\left(X,{ }^{b} \Omega^{\frac{1}{2}}\right)\right.$ for a resulting asymptotic type $Q$ depending on $a$ and $Q^{\prime}$ ).

The cone algebra $\mathfrak{C}^{m}\left(X,{ }^{b} \Omega^{\frac{1}{2}},(\gamma, \gamma-m, k)\right)$ is spectrally invariant, i.e. if an element $a$ of this class is invertible as an operator $\mathcal{H}^{s, \gamma}\left(X,{ }^{b} \Omega^{\frac{1}{2}}\right) \rightarrow \mathcal{H}^{s-m, \gamma-m}\left(X,{ }^{b} \Omega^{\frac{1}{2}}\right)$ for some $s$, the inverse $a^{-1}$ belongs to $\mathfrak{C}^{-m}\left(X,{ }^{b} \Omega^{\frac{1}{2}},(\gamma-m, \gamma, k)\right)$.

REMARK 3.10. From general results on the invertibility of meromorphic Fredholm families it can be deduced that if a Mellin symbol in $M_{P}^{m}\left(\partial X, \Omega^{\frac{1}{2}}\right)$ is invertible on a vertical line, its inverse automatically has the same structure, cf. [58], Theorem 2.4.20. However, this is not true for symbols of weak type. Hence, for the weak cone algebra analogous mapping properties are valid, but the invertibility of both principal symbols is not sufficient to ensure the existence of a parametrix within the class. In addition, one has to require that, with the above notation, $h(0)+h_{0}$ is meromorphically invertible within the weak class.

3.6. Further remarks on the cone algebra. We consider the relation of the cone algebras with respect to finite weight intervals and those for infinite intervals.

Proposition 3.11. The following identity is valid:

$$
\bigcap_{k \in \mathbb{N}} \mathfrak{C}^{m-j}\left(X,{ }^{b} \Omega^{\frac{1}{2}},(\gamma, \gamma-m, k)\right)=\mathfrak{C}^{m-j}\left(X,{ }^{b} \Omega^{\frac{1}{2}},(\gamma, \gamma-m, \infty)\right) .
$$

Proof. Obviously the right-hand side is contained in the left one. Now let $a$ have for each $k$ a representation $a=h_{k}+a_{\psi}^{k}+M_{k}+G_{k}$ with the obvious meaning of notation. Then $h_{k}+a_{\psi}^{k}-\left(h_{0}+a_{\psi}^{0}\right)$ is an element of

$$
\bigcap_{l \in \mathbb{N}} \underbrace{\mathfrak{C}^{m-j}\left(X,{ }^{b} \Omega^{\frac{1}{2}},(\gamma, \gamma-m, l)\right) \cap \Psi^{-\infty}\left(X_{0}, \Omega^{\frac{1}{2}}\right)}_{=\mathfrak{C}_{M+G}^{m-j}\left(X,{ }^{b} \Omega^{\frac{1}{2}},(\gamma, \gamma-m, l)\right)}=\mathfrak{C}_{M+G}^{m-j}\left(X,{ }^{b} \Omega^{\frac{1}{2}},(\gamma, \gamma-m, \infty)\right) .
$$

For the '=' under the bracket see for instance [4], Chapter 8.1.4, Remark 2. Hence $a-\left(h_{0}+a_{\psi}^{0}\right)=\left(h_{k}+a_{\psi}^{k}-\left(h_{0}+a_{\psi}^{0}\right)\right)+M_{k}+G_{k} \in \mathfrak{C}_{M+G}^{m-j}\left(X,{ }^{b} \Omega^{\frac{1}{2}},(\gamma, \gamma-m, k)\right)$.

Since this is true for any $k$, we obtain $a=h_{0}+a_{\psi}^{0}$ modulo $\mathfrak{C}_{M+G}^{m-j}\left(X,{ }^{b} \Omega^{\frac{1}{2}},(\gamma, \gamma-\right.$ $m, \infty))$.

As a provisional notation, let us introduce $\mathfrak{C}_{M+G}^{m-j}\left(X,{ }^{b} \Omega^{\frac{1}{2}},(\gamma, \gamma-m, k)\right)_{O}, k \in$ $\mathbb{N} \cup\{\infty\}$, as the space of operators $M+G$ as in Definition 3.7 , but with all $h_{l}$ holomorphic and $G \in \mathfrak{C}_{G}\left(X,{ }^{b} \Omega^{\frac{1}{2}},(\gamma, \gamma-m, k)\right)_{O, O}$. Using these remainders, we define $\mathfrak{C}^{m-j}\left(X,{ }^{b} \Omega^{\frac{1}{2}},(\gamma, \gamma-m, k)\right)_{O}$ analogously as in Definition 3.8. For $k<\infty$ it is obvious that this definition coincides with that given in Definition 3.9. This is also true for $k=\infty$, as we shall show in Proposition 3.13 below. 
LEMMA 3.12. An operator a belongs to $\mathfrak{C}_{M+G}^{m-j}\left(X,{ }^{b} \Omega^{\frac{1}{2}},(\gamma, \gamma-m, \infty)\right)_{O}$ if and only if

$$
a=\omega_{0} x^{m-j} \operatorname{op}_{M}^{\left(\gamma-\frac{n-1}{2}\right)}\left(h_{\infty}\right) \omega_{1} \bmod \mathfrak{C}_{G}^{m-j}\left(X,{ }^{b} \Omega^{\frac{1}{2}},(\gamma, \gamma-m, \infty)\right)_{O}
$$

for a certain $h_{\infty} \in \mathcal{C}^{\infty}\left(\overline{\mathbb{R}}_{+}, M_{O}^{-\infty}\left(\partial X, \Omega^{\frac{1}{2}}\right)\right)$.

Proof. If $a$ is represented in the described manner, it belongs for each $k$ to $\mathfrak{C}_{M+G}^{m-j}\left(X,{ }^{b} \Omega^{\frac{1}{2}},(\gamma, \gamma-m, k)\right)_{O}$ simply by Taylor expansion of $h$ in $x$ at 0 . Vice versa, if $a \in \mathfrak{C}_{M+G}^{m-j}\left(X,{ }^{b} \Omega^{\frac{1}{2}},(\gamma, \gamma-m, \infty)\right)_{O}$ there exists a unique sequence $\left(h_{l}\right)_{l \in \mathbb{N}_{0}}$ in $M_{O}^{-\infty}\left(\partial X, \Omega^{\frac{1}{2}}\right)$ (the lower conormal symbols of $a$ ), such that

$$
a=\omega_{0}\left\{\sum_{l=0}^{k-j-1} x^{-m+j+l} \operatorname{op}_{M}^{\left(\gamma_{l}-\frac{n-1}{2}\right)}\left(h_{l}\right)\right\} \omega_{1} \bmod \mathfrak{C}_{G}^{m-j}\left(X,{ }^{b} \Omega^{\frac{1}{2}},(\gamma, \gamma-m, k)\right)_{O}
$$

for any $k$. By Borel theorem, there exists an $h_{\infty} \in \mathcal{C}^{\infty}\left(\overline{\mathbb{R}}_{+}, M_{O}^{-\infty}\left(\partial X, \Omega^{\frac{1}{2}}\right)\right)$ such that $h_{\infty}^{(l)}(0)=l ! h_{l}$ for all $l$. This $h_{\infty}$ is as required.

Proposition 3.13. The following identity is valid:

$$
\bigcap_{k \in \mathbb{N}} \mathfrak{C}^{m-j}\left(X,{ }^{b} \Omega^{\frac{1}{2}},(\gamma, \gamma-m, k)\right)_{O}=\mathfrak{C}^{m-j}\left(X,{ }^{b} \Omega^{\frac{1}{2}},(\gamma, \gamma-m, \infty)\right)_{O} .
$$

Proof. Again the right-hand side is contained in the left one. If $a$ belongs to the left-hand side, then with obvious meaning of notation, $a=h_{0}+a_{\psi}^{0}+R$ with $R \in \mathfrak{C}_{M+G}^{m-j}\left(X,{ }^{b} \Omega^{\frac{1}{2}},(\gamma, \gamma-m, \infty)\right)_{O}$ by Proposition 3.11 . However, by Lemma 3.12 , $R$ can be represented as $R=h_{\infty}+G$ with $G \in \mathfrak{C}_{G}^{m-j}\left(X,{ }^{b} \Omega^{\frac{1}{2}},(\gamma, \gamma-m, \infty)\right)_{O}$. But then $a=\left(h_{0}+h_{\infty}\right)+a_{\psi}^{0}+G$ is an element of $\mathfrak{C}^{m-j}\left(X,{ }^{b} \Omega^{\frac{1}{2}},(\gamma, \gamma-m, \infty)\right)_{O}$.

\section{Review on the (overblown) b-calculus}

4.1. Polyhomogeneous conormal functions. We are going to introduce subspaces of conormal functions that have a certain behavior at the boundary hyperfaces of $Z$. To capture this behavior we need the notion of an index set $[\mathbf{3 1}$, Definition 5.20].

Definition 4.1. A discrete subset $E \subseteq \mathbb{C} \times \mathbb{N}_{0}$ is said to be an absolute index set provided

1. $\left(z_{j}, k_{j}\right) \in E$ with $\left|\left(z_{j}, k_{j}\right)\right| \rightarrow \infty \Longrightarrow \operatorname{Re}\left(z_{j}\right) \rightarrow \infty$,

2. $(z, k) \in E \Longrightarrow(z, \ell) \in E$ for all $\ell \in \mathbb{N}_{0}$ with $0 \leq \ell \leq k$.

An absolute index set satisfying

3. $(z, k) \in E \Longrightarrow(z+j, k) \in E$ for all $j \in \mathbb{N}_{0}$,

is called a $\mathcal{C}^{\infty}$-index set or simply an index set. For any index set $E$, let

$$
\inf E:=\min \{\operatorname{Re}(z):(z, 0) \in E\} .
$$

An index family $\mathcal{E}$ for a manifold $Z$ with corners is a map $\mathcal{E}=\left(E_{H}\right)_{H \in \mathcal{F}_{1}(Z)}$ that associates to each $H \in \mathcal{F}_{1}(Z)$ a $\mathcal{C}^{\infty}$-index set $E_{H}=\mathcal{E}(H)$. 
We agree to write 0 for the special index set $\left\{(\ell, 0) \in \mathbb{C} \times \mathbb{N}_{0}: \ell \in \mathbb{N}_{0}\right\}$. Fix $G \in \mathcal{F}_{1}(Z)$, and let $\mathcal{E}_{G}(H):=0$ for $G \neq H \in \mathcal{F}_{1}(Z)$ and $\mathcal{E}_{G}(G)=E_{G}$ for some $\mathcal{C}^{\infty}$-index set $E_{G}$. Furthermore, let us denote by $\dot{\mathcal{C}}_{G}^{N}(Z)$ the space of all $N$-times differentiable functions that vanish up to order $N$ at $G$.

Definition 4.2. The space $\mathcal{A}_{p h g}^{\mathcal{E}_{G}}(Z)$ consists of all $u \in \mathcal{A}(Z)$ for which there exist $a_{z, k} \in \mathcal{C}^{\infty}(Z)$ with

$$
u-\sum_{(z, k) \in E_{G}, \operatorname{Re}(z) \leq N} \varrho_{G}^{z}\left(\log \varrho_{G}\right)^{k} a_{z, k} \in \dot{\mathcal{C}}_{G}^{N}(Z)
$$

for all $N \in \mathbb{N}_{0}$. The elements of $\mathcal{A}_{p h g}^{\mathcal{E}_{G}}(Z)$ are called polyhomogeneous at $G$.

Clearly, the definition does not depend on the choice of the defining function $\varrho_{G}$ of $G$. Note that for $E_{G}=0$ we have $\mathcal{A}_{p h g}^{\mathcal{E}_{G}}(Z)=\mathcal{C}^{\infty}(Z)$ by Taylor's theorem. A Fréchet topology on $\mathcal{A}_{p h g}^{\mathcal{E}_{G}}(Z)$ is given by the $\mathcal{C}^{N}$-topology for the remainders in (4.1), and the $\mathcal{C}^{\infty}(Z)$-topology for the coefficients $a_{z, k} \in \mathcal{C}^{\infty}(Z)$.

Definition 4.2 can easily be generalized to index families $\mathcal{E}=\left(E_{F}\right)_{F \in \mathcal{F}_{1}(Z)}$ satisfying

$$
E_{G} \neq 0 \text { and } E_{F} \neq 0 \Longrightarrow G \cap F=\emptyset .
$$

Indeed, choose a partition of unity $\sum_{\mathcal{E}(G) \neq 0} \varphi_{G}=1$ on $Z$ with $\operatorname{supp} \varphi_{G} \cap F=\emptyset$ for all $F, G \in \mathcal{F}_{1}(Z)$ with $\mathcal{E}(F) \neq 0, \mathcal{E}(G) \neq 0$, and $F \neq G$.

Definition 4.3. Let $\mathcal{E}$ be an index family with the separation property (4.2). Then the space $\mathcal{A}_{p h g}^{\mathcal{E}}(Z)$ of polyhomogeneous conormal functions with index family $\mathcal{E}$ consists of all $u \in \mathcal{A}(Z)$ with $\varphi_{G} u \in \mathcal{A}_{p h g}^{\mathcal{E}_{G}}(Z)$ for all $G \in \mathcal{F}_{1}(Z)$ with $\mathcal{E}(G) \neq 0$. Here $\mathcal{E}_{G}(F)=0$ for $G \neq F$ and $\mathcal{E}_{G}(G)=\mathcal{E}(G)$.

The Fréchet topology on the spaces $\mathcal{A}_{p h g}^{\mathcal{E}_{G}}(Z)$ for $G \in \mathcal{F}_{1}(Z)$ with $\mathcal{E}(G) \neq 0$ induces a Fréchet topology on $\mathcal{A}_{p h g}^{\mathcal{E}}(Z)$. Let us mention that in the special case $\mathcal{E}(H)=\emptyset$ any $u \in \mathcal{A}_{p h g}^{\mathcal{E}}(Z)$ vanishes with all derivatives at $H \in \mathcal{F}_{1}(Z)$. This follows easily from the expansion (4.1).

Since $\mathcal{A}_{p h g}^{\mathcal{E}}(Z)$ is a $\mathcal{C}^{\infty}(Z)$-module, we can define for any smooth vector bundle $F \rightarrow Z$

$$
\mathcal{A}_{p h g}^{\mathcal{E}}(Z, F):=\mathcal{A}_{p h g}^{\mathcal{E}}(Z) \otimes_{\mathcal{C} \infty(Z)} \mathcal{C}^{\infty}(Z, F) .
$$

Moreover, if $\mathcal{E}, \mathcal{F}$, and $\mathcal{E} \cup \mathcal{F}$ are index families for $Z$ satisfying the separation condition (4.2) then we have [26, (A.10)]

$$
\mathcal{A}_{p h g}^{\mathcal{E}}(Z, F)+\mathcal{A}_{p h g}^{\mathcal{F}}(Z, F)=\mathcal{A}_{p h g}^{\mathcal{E} \cup \mathcal{F}}(Z, F)
$$

However, later on we need the notion of the extended union of two index sets $E$ and $F$. It is defined by $[\mathbf{3 1},(5.120)]$

$$
E \bar{\cup} F:=E \cup F \cup\left\{(z, k) \in \mathbb{C} \times \mathbb{N}_{0}: \exists\left(z, \ell_{1}\right) \in E, \exists\left(z, \ell_{2}\right) \in F: k=\ell_{1}+\ell_{2}+1\right\} \text {. }
$$


In fact, it is possible to define the spaces $\mathcal{A}_{p h g}^{\mathcal{E}}(Z)$ or $\mathcal{A}_{p h g}^{\mathcal{E}}(Z, F)$ for arbitrary index families by induction with respect to the codimension of $Z$; we refer the reader to [5], [27], or [35] for the details. Instead, we concentrate on the special case $Z=X^{2}[\mathbf{3 1}$, Definition 5.49] where $X$ is a compact manifold with boundary because this is the only case beyond Definition 4.3 we really need.

Let $\mathcal{E}, \mathcal{F}$ be index families for $X$, let $\left(\varphi_{H}\right)_{H \in \mathcal{F}_{1}(X)}$ be a partition of unity with $H \subseteq \operatorname{supp} \varphi_{H}$ and $G \cap \operatorname{supp} \varphi_{H}=\emptyset$ for all $G, H \in \mathcal{F}_{1}(X)$ with $G \neq H$, and let $\varrho_{H}: X \longrightarrow \overline{\mathbb{R}}_{+}$be a defining function for $H \in \mathcal{F}_{1}(X)$. Moreover, let $(0, \mathcal{F})$ be the index family on $X^{2}$ given by $(0, \mathcal{F})(X \times H)=\mathcal{F}(H)$ and $(0, \mathcal{F})(H \times X)=0$

Definition 4.4. The space $\mathcal{A}_{p h g}^{(\mathcal{E}, \mathcal{F})}\left(X^{2}\right)$ consists of those conormal functions $u \in \mathcal{A}\left(X^{2}\right)$ for which for any $H \in \mathcal{F}_{1}(X)$ there exist $a_{z, k}^{(H)} \in \mathcal{A}_{p h g}^{(0, \mathcal{F})}\left(X^{2}\right)$ satisfying

$$
\varphi_{H} u-\sum_{(z, k) \in \mathcal{E}(H), \operatorname{Re}(z) \leq N} \varrho_{H}^{z} \log ^{k} \varrho_{H} a_{z, k}^{(H)} \in \dot{\mathcal{C}}_{H}^{N}\left(X, \mathcal{A}_{p h g}^{\mathcal{F}}(X)\right)
$$

for all $N \in \mathbb{N}_{0}$. Here $\dot{\mathcal{C}}_{H}^{N}\left(X, \mathcal{A}_{p h g}^{\mathcal{F}}(X)\right)$ is the space of all $N$-times differentiable functions $X \longrightarrow \mathcal{A}_{p h g}^{\mathcal{F}}(X)$ that vanish up to order $N$ at $H$.

Let us stress the fact that this definition is independent of the ordering and invariant under diffeomorphism - for the details we refer the reader to [35]. The vector valued version can be defined for any smooth vector bundle $F \longrightarrow X^{2}$ by $\mathcal{A}_{p h g}^{(\mathcal{E}, \mathcal{F})}\left(X^{2}, F\right):=\mathcal{A}_{p h g}^{(\mathcal{E}, \mathcal{F})}\left(X^{2}\right) \otimes_{\mathcal{C} \infty\left(X^{2}\right)} \mathcal{C}^{\infty}\left(X^{2}, F\right)$.

We are now going to consider the behavior of the Mellin transform of conormal functions $\overline{\mathbb{R}}_{+} \times Z \longrightarrow \mathbb{C}$ that are polyhomogeneous at $\{0\} \times Z$. To get rid of the problems with the non-compactness of $\overline{\mathbb{R}}_{+}$at infinity, we use the diffeomorphism $\mathbb{R}_{+} \longrightarrow(-1,1): x \longmapsto \tau=\frac{x-1}{x+1}$. Under this map, the space $\mathcal{A}^{\mathcal{E}}([-1,1])$ with $\mathcal{E}(\{-1\})=E$ and $\mathcal{E}(\{1\})=\emptyset$ for some $\mathcal{C}^{\infty}$-index set $E$ correspond to the space of smooth functions $\mathbb{R}_{+} \longrightarrow \mathbb{C}$ having the polyhomogeneous conormal expansion given by $E$ at 0 , and vanishing rapidly at $\infty$. For a proof of the following characterization we refer to [31, Proposition 5.27] - note that we changed the definition of the Mellin transform by a factor $-i$.

Proposition 4.5. Let $Z$ be a compact manifold with corners, $E$ a $\mathcal{C}^{\infty}$-index set, and $\mathcal{E}$ the index family for $[-1,1] \times Z$ with $\mathcal{E}(\{-1\} \times Z)=E, \mathcal{E}(\{1\} \times Z)=\emptyset$, and $\mathcal{E}([-1,1] \times H)=0$ for all $H \in \mathcal{F}_{1}(Z)$. Then the Mellin transform

$$
M u: \mathbb{C} \times Z \ni(z, p) \longmapsto \int_{0}^{\infty} x^{z} u\left(\frac{x-1}{x+1}, p\right) \frac{d x}{x}=2 \int_{-1}^{1}\left(\frac{1+\tau}{1-\tau}\right)^{z} u(\tau, p) \frac{d \tau}{1-\tau^{2}}
$$

induces an isomorphism from the space $\mathcal{A}^{\mathcal{E}}([-1,1] \times Z)$ onto the space of all meromorphic functions $\mathbb{C} \longrightarrow \mathcal{C}^{\infty}(Z)$ having poles of order $k+1$ only at points $-z$ with $(z, k) \in E$ and satisfying for each large $N \in \mathbb{N}$

$$
\|\left. M u(z, \cdot)\right|_{\mathcal{C}^{N}(Z)} \leq C_{N}<|z|>^{-N} \text { in }|\operatorname{Re}(z)| \leq C_{N} \text { and }|\operatorname{Im}(z)| \geq C_{N}
$$

for some $C_{N}>0$. Here $\|\cdot\|_{\mathcal{C}^{N}(Z)}$ is a norm on the Banach space $\mathcal{C}^{N}(Z)$ of all $N$-times differentiable functions on $Z$. 
4.2. The full b-calculus: definition and basic properties. From now on, let $X$ be a compact manifold of dimension $n$ with boundary $\partial X$. Recall that by the Schwartz kernel theorem there is a one-to-one correspondence between continuous linear operators $a: \dot{\mathcal{C}}^{\infty}\left(X,{ }^{b} \Omega^{\frac{1}{2}}\right) \longrightarrow \mathcal{C}^{-\infty}\left(X,{ }^{b} \Omega^{\frac{1}{2}}\right)$ and their Schwartz kernels $k_{a} \in \mathcal{C}^{-\infty}\left(X^{2},{ }^{b} \Omega^{\frac{1}{2}}\right)$. However, to get a convenient description of the Schwartz kernels of b-pseudodifferential operators on $X$ we have to modify the manifold $X^{2}$ by blowing up the p-submanifold

$$
B:=\underset{H \in \mathcal{F}_{1}(X)}{\cup} H \times H \subseteq(\partial X)^{2} .
$$

For the general concept of blowing up which essentially corresponds to the introduction of polar coordinates we refer the reader to $[\mathbf{2 7}],[\mathbf{3 1}]$, [32], or [35].

Proposition 4.6. Let $X_{b}^{2}:=\left[X^{2} ; B\right]$ be the compact manifold with corners obtained by blowing up $X^{2}$ along the p-submanifold $B \subseteq(\partial X)^{2}$. Then the blow down $\operatorname{map} \beta_{b}^{2}: X_{b}^{2} \longrightarrow X^{2}$ induces isomorphisms

$$
\begin{aligned}
\left(\beta_{b}^{2}\right)^{*}: \dot{\mathcal{C}}^{\infty}\left(X^{2},{ }^{b} \Omega^{\frac{1}{2}}\right) & \stackrel{\cong}{\longrightarrow} \dot{\mathcal{C}}^{\infty}\left(X_{b}^{2},{ }^{b} \Omega^{\frac{1}{2}}\right), \text { and } \\
\left(\beta_{b}^{2}\right)_{*}: \mathcal{C}^{-\infty}\left(X_{b}^{2},{ }^{b} \Omega^{\frac{1}{2}}\right) & \cong \mathcal{C}^{-\infty}\left(X^{2},{ }^{b} \Omega^{\frac{1}{2}}\right) .
\end{aligned}
$$

The lifted diagonal $\Delta_{b}:={\overline{\left(\beta_{b}^{2}\right)^{-1}(\Delta \backslash B)}}^{X_{b}^{2}}$ meets the boundary $\partial X_{b}^{2}$ only in the faces $\mathrm{ff}^{b}(H):=\left(\beta_{b}^{2}\right)^{-1}(H \times H), H \in \mathcal{F}_{1}(X)$, and intersects them transversally. The submanifold $\mathrm{ff}^{b}:=\bigcup_{H \in \mathcal{F}_{1}(X)} \mathrm{ff}^{b}(H)$ is called the front face of $X_{b}^{2}$.

Let us label also the other faces of $X_{b}^{2}$. For each boundary face $H \in \mathcal{F}_{1}(X)$, let $\operatorname{lb}(H):={\overline{\left(\beta_{b}^{2}\right)^{-1}((H \times X) \backslash B)}}^{X_{b}^{2}}$ and $\operatorname{rb}(H):={\overline{\left(\beta_{b}^{2}\right)^{-1}((X \times H) \backslash B)}}^{X_{b}^{2}}$. The submanifolds $\mathrm{lb}:=\bigcup_{H \in \mathcal{F}_{1}(X)} \mathrm{lb}(H)$ resp. $\mathrm{rb}:=\bigcup_{H \in \mathcal{F}_{1}(X)} \mathrm{rb}(H)$ are called the left resp. right boundary of $X_{b}^{2}$.

The important point to note here is that $\mathcal{C}^{\infty}\left(X^{2},{ }^{b} \Omega^{\frac{1}{2}}\right) \not \subset\left(\beta_{b}^{2}\right)_{*}\left(\mathcal{C}^{\infty}\left(X_{b}^{2},{ }^{b} \Omega^{\frac{1}{2}}\right)\right)$, i.e. there are "more" $\mathcal{C}^{\infty}$-functions on $X_{b}^{2}$ than on $X^{2}$ which is the flexibility needed for an appropriate description of the kernels of b-pseudodifferential operators. Let us denote by $\kappa_{a} \in \mathcal{C}^{-\infty}\left(X_{b}^{2},{ }^{b} \Omega^{\frac{1}{2}}\right)$ the (lifted) Schwartz kernel corresponding to $a: \dot{\mathcal{C}}^{\infty}\left(X,{ }^{b} \Omega^{\frac{1}{2}}\right) \longrightarrow \mathcal{C}^{-\infty}\left(X,{ }^{b} \Omega^{\frac{1}{2}}\right)$. For the next definition see [31, Definition $4.22]$.

Definition 4.7. A continuous linear map $a: \dot{\mathcal{C}}^{\infty}\left(X,{ }^{b} \Omega^{\frac{1}{2}}\right) \longrightarrow \mathcal{C}^{-\infty}\left(X,{ }^{b} \Omega^{\frac{1}{2}}\right)$ belongs to the space $\Psi_{b, c l}^{m}\left(X,{ }^{b} \Omega^{\frac{1}{2}}\right)$ of classical, b-pseudodifferential operators of order $m \in \mathbb{R}$ provided

$$
\kappa_{a} \in\left\{\kappa \in I_{c l}^{m}\left(X_{b}^{2}, \Delta_{b} ;{ }^{b} \Omega^{\frac{1}{2}}\right): \kappa \equiv 0 \text { at } \partial X_{b}^{2} \backslash \mathrm{ff}^{b}\right\} \subseteq \mathcal{C}^{-\infty}\left(X_{b}^{2},{ }^{b} \Omega^{\frac{1}{2}}\right) .
$$

Here $I_{c l}^{m}\left(X_{b}^{2}, \Delta_{b} ;{ }^{b} \Omega^{\frac{1}{2}}\right)$ denotes the space of classical conormal distributions as introduced in $[\mathbf{1 0}]$ - see also $\left[\mathbf{1 1}\right.$, Section 18.2]. The spaces $\Psi_{b, c l}^{m}\left(X,{ }^{b} \Omega^{\frac{1}{2}}\right), m \in \mathbb{R}$, are called the small b-calculus. 
The same definition extends to the case of compact manifolds with corners see [22], [39]. Note that the restriction to classical conormal distributions in Definition 4.7 is not really necessary; however, it guarantees a nice symbolic structure.

Since there is a canonical identification of the conormal bundle $N^{*} \Delta_{b}$ of the lifted diagonal $\Delta_{b} \subseteq X_{b}^{2}$ and the b-cotangent bundle ${ }^{b} T^{*} X$, the homogeneous principal symbol for classical conormal distributions [10] (see also [11]) gives for each $m \in \mathbb{R}$ a map ${ }^{b} \sigma^{(m)}: \Psi_{b, c l}^{m}\left(X,{ }^{b} \Omega^{\frac{1}{2}}\right) \longrightarrow S^{[m]}\left({ }^{b} T^{*} X\right)$, where the latter is the space of smooth functions ${ }^{b} T^{*} X \backslash\{0\} \longrightarrow \mathbb{C}$ that are homogeneous of degree $m$ in the fibers. If we choose a defining function $\varrho_{\sigma}$ of the $b$-cosphere-bundle ${ }^{b} S^{*} X:=\left({ }^{b} T^{*} X \backslash\{0\}\right) / \mathbb{R}_{+}$in the radial compactification of ${ }^{b} T^{*} X$, we can identify $S^{[m]}\left({ }^{b} T^{*} X\right)$ with $\mathcal{C}^{\infty}\left({ }^{b} S^{*} X\right)$, and the homogeneous principal symbol induces the following short exact sequence

$$
0 \longrightarrow \Psi_{b, c l}^{m-1}\left(X,{ }^{b} \Omega^{\frac{1}{2}}\right) \longrightarrow \Psi_{b, c l}^{m}\left(X,{ }^{b} \Omega^{\frac{1}{2}}\right) \stackrel{{ }^{b}}{\sigma^{(m)}} \mathcal{C}^{\infty}\left({ }^{b} S^{*} X\right) \longrightarrow 0 .
$$

To capture the behavior of b-pseudodifferential operators at the boundary, let $G \in \mathcal{F}_{1}(X)$ be a component of the boundary, $\varrho_{G}: X \longrightarrow \overline{\mathbb{R}}_{+}$be a defining function for $G$, and $(\cdot)_{G}: \Psi_{b, c l}^{m}\left(X,{ }^{b} \Omega^{\frac{1}{2}}\right) \longrightarrow \Psi_{c l}^{m}\left(G, \Omega^{\frac{1}{2}}\right)$ be the morphism of restricting to $G$. The map

$$
I_{\varrho G}^{(G)}: \Psi_{b, c l}^{m}\left(X,{ }^{b} \Omega^{\frac{1}{2}}\right) \longrightarrow M_{\mathcal{O}}^{m}\left(G, \Omega^{\frac{1}{2}}\right): a \longmapsto\left[z \longmapsto\left(\varrho_{G}^{z} a \varrho_{G}^{-z}\right)_{G}\right]
$$

is called the indicial family at $G$ - cf. [31, Proposition 5.3], [18, Proposition 2.3.1]. The homogeneous principal symbol and the indicial family allow to characterize the Fredholm operators in the small calculus. [36, Theorem 6.17]- see also [22], $[31],[39]$, or $[41]$.

Theorem 4.8. Let $a \in \Psi_{b, c l}^{m}\left(X,{ }^{b} \Omega^{\frac{1}{2}}\right)$, and $\mathfrak{b}_{G} \in \mathbb{R}, G \in \mathcal{F}_{1}(X)$ be an arbitrary weight system. Then $a: \dot{\mathcal{C}}^{\infty}\left(X,{ }^{b} \Omega^{\frac{1}{2}}\right) \longrightarrow \dot{\mathcal{C}}^{\infty}\left(X,{ }^{b} \Omega^{\frac{1}{2}}\right)$ extends for each $s \in \mathbb{R}$ to a bounded operator $a: \varrho^{\mathfrak{b}} H_{b}^{s}\left(X,{ }^{b} \Omega^{\frac{1}{2}}\right) \longrightarrow \varrho^{\mathfrak{b}} H_{b}^{s-m}\left(X,{ }^{b} \Omega^{\frac{1}{2}}\right)$ which is Fredholm if and only if $I_{\varrho_{G}}^{G}(a)\left(i \lambda-\mathfrak{b}_{G}\right): H^{s}\left(G, \Omega^{\frac{1}{2}}\right) \longrightarrow H^{s-m}\left(G, \Omega^{\frac{1}{2}}\right)$ is invertible for all $\lambda \in \mathbb{R}$ and all $G \in \mathcal{F}_{1}(X)$, and ${ }^{b} \sigma^{(m)}(a)(\zeta) \neq 0$ for all $\zeta \in{ }^{b} S^{*} X$.

To understand the nature of the generalized inverses of Fredholm operators in the small calculus we have to enlarge the small b-calculus by allowing nontrivial expansions at the left resp. right boundary. For simplicity, let us assume that the boundary $\partial X$ is connected. Then we have $\mathcal{F}_{1}\left(X_{b}^{2}\right)=\left\{\mathrm{lb}, \mathrm{rb}, \mathrm{ff}^{b}\right\}$ with $\mathrm{lb} \cap \mathrm{rb}=\emptyset$. Let $E_{\mathrm{lb}}$ resp. $E_{\mathrm{rb}}$ be $\mathcal{C}^{\infty}$-index sets, and denote by $\mathcal{E}$ the index family $\left(E_{\mathrm{lb}}, E_{\mathrm{rb}}, 0\right)$ corresponding to the ordering (lb, rb, $\left.\mathrm{ff}^{b}\right)$ of $\mathcal{F}_{1}\left(X_{b}^{2}\right)$ - note that $\mathcal{E}$ satisfies the separation property (4.2), and we can define $\widetilde{\Psi}_{b}^{-\infty, \mathcal{E}}\left(X,{ }^{b} \Omega^{\frac{1}{2}}\right)$ as the space of all $a: \dot{\mathcal{C}}^{\infty}\left(X,{ }^{b} \Omega^{\frac{1}{2}}\right) \longrightarrow \mathcal{C}^{-\infty}\left(X,{ }^{b} \Omega^{\frac{1}{2}}\right)$ with $\kappa_{a} \in \mathcal{A}_{p h g}^{\mathcal{E}}\left(X_{b}^{2},{ }^{b} \Omega^{\frac{1}{2}}\right)[\mathbf{3 1}$, (5.82)], and $\Psi^{-\infty, \mathcal{E}}\left(X,{ }^{b} \Omega^{\frac{1}{2}}\right)$ as that of all $a: \dot{\mathcal{C}}^{\infty}\left(X,{ }^{b} \Omega^{\frac{1}{2}}\right) \longrightarrow \mathcal{C}^{-\infty}\left(X,{ }^{b} \Omega^{\frac{1}{2}}\right)$ with Schwartz kernel $k_{a} \in \mathcal{A}_{p h g}^{\left(E_{\mathrm{lb}}, E_{\mathrm{rb}}\right)}\left(X^{2},{ }^{b} \Omega^{\frac{1}{2}}\right)$, where $\left(E_{\mathrm{lb}}, E_{\mathrm{rb}}\right)$ is the index family on $X^{2}$ corresponding to the left $\mathrm{lb}=\partial X \times X$ resp. right boundary $\mathrm{rb}=X \times \partial X$ 
of $X^{2}$ [31, p. 198]. We are now ready to define the full b-calculus [31, Definition $5.51]$.

DEFinition 4.9. The full calculus of classical, b-pseudodifferential operators of order $m \in \mathbb{R}$ with respect to the index family $\mathcal{E}=\left(E_{\mathrm{lb}}, E_{\mathrm{rb}}\right)$ is the space

$$
\Psi_{b, c l}^{m, \mathcal{E}}\left(X,{ }^{b} \Omega^{\frac{1}{2}}\right):=\Psi_{b, c l}^{m}\left(X,{ }^{b} \Omega^{\frac{1}{2}}\right)+\widetilde{\Psi}_{b}^{-\infty, \mathcal{E}}\left(X,{ }^{b} \Omega^{\frac{1}{2}}\right)+\Psi^{-\infty, \mathcal{E}}\left(X,{ }^{b} \Omega^{\frac{1}{2}}\right) .
$$

Note that $\Psi_{b, c l}^{*, \mathcal{E}}\left(X,{ }^{b} \Omega^{\frac{1}{2}}\right)$ is called the full-calculus and not the full-algebra because composition is not always possible. However, for any two index families $\mathcal{E}=\left(E_{\mathrm{lb}}, E_{\mathrm{rb}}\right)$ and $\mathcal{F}=\left(F_{\mathrm{lb}}, F_{\mathrm{rb}}\right)$ with inf $E_{\mathrm{rb}}+\inf F_{\mathrm{lb}}>0$ we have [31, Theorem $5.53]$

$$
\Psi_{b, c l}^{m, \mathcal{E}}\left(X,{ }^{b} \Omega^{\frac{1}{2}}\right) \circ \Psi_{b, c l}^{m^{\prime}, \mathcal{F}}\left(X,{ }^{b} \Omega^{\frac{1}{2}}\right) \subseteq \Psi_{b, c l}^{m+m^{\prime}, \mathcal{G}}\left(X,{ }^{b} \Omega^{\frac{1}{2}}\right)
$$

with $\mathcal{G}(\mathrm{lb})=E_{\mathrm{lb}} \bar{\cup} F_{\mathrm{lb}}$ and $\mathcal{G}(\mathrm{rb})=E_{\mathrm{rb}} \bar{\cup} F_{\mathrm{rb}}$. Moreover, $a \in \Psi_{b, c l}^{m, \mathcal{E}}\left(X,{ }^{b} \Omega^{\frac{1}{2}}\right)$ induces a bounded operator [31, Theorem 5.34]

$$
a: \varrho^{\mathfrak{b}} H_{b}^{s}\left(X,{ }^{b} \Omega^{\frac{1}{2}}\right) \longrightarrow \varrho^{\mathfrak{b}^{\prime}} H_{b}^{s-m}\left(X,{ }^{b} \Omega^{\frac{1}{2}}\right) \text { provided }\left\{\begin{array}{l}
\mathfrak{b}^{\prime} \leq \mathfrak{b}, \\
\mathfrak{b}+\inf E_{\mathrm{rb}}>0 \\
\mathfrak{b}^{\prime}<\inf E_{\mathrm{lb}} .
\end{array}\right.
$$

If $a \in \Psi_{b, c l}^{m}\left(X,{ }^{b} \Omega^{\frac{1}{2}}\right)$ is a Fredholm operator $a: \varrho^{\mathfrak{b}} H_{b}^{m}\left(X,{ }^{b} \Omega^{\frac{1}{2}}\right) \longrightarrow \varrho^{\mathfrak{b}} L^{2}\left(X,{ }^{b} \Omega^{\frac{1}{2}}\right)$, then, by [31, Proposition 5.64], the generalized inverse $b$ of $a$, i.e. the operator $b: \varrho^{\mathfrak{b}} L^{2}\left(X,{ }^{b} \Omega^{\frac{1}{2}}\right) \longrightarrow \varrho^{\mathfrak{b}} H_{b}^{m}\left(X,{ }^{b} \Omega^{\frac{1}{2}}\right)$ inverting $a$ up to the orthogonal projection onto the kernel and cokernel, belongs to $\Psi_{b, c l}^{-m, \mathcal{E}}\left(X,{ }^{b} \Omega^{\frac{1}{2}}\right)$. Here $\mathcal{E}$ is an index family depending on the roots of the indicial family and $\mathfrak{b} \in \mathbb{R}$.

4.3. The overblown b-calculus. Recall that we defined the b-double space $X_{b}^{2}$ by blowing up those components of $(\partial X)^{2}$ intersecting the diagonal. By blowing up the whole corner $(\partial X)^{2}$ we obtain a compact manifold with corners that is known as the overblown b-double space

$$
\beta_{o b}^{2}: X_{o b}^{2}:=\left[X^{2},(\partial X)^{2}\right] \longrightarrow X^{2} .
$$

Of course, we have $X_{o b}^{2}=X_{b}^{2}$ if $\partial X$ is connected. Exactly as in Subsection 4.2 we can define the corresponding overblown b-pseudodifferential calculus. This calculus naturally occurs as the range of the b-normal homomorphism in the surgery calculus [27, Section 4.6]. For simplicity, we restrict ourselves to the definition of the small calculus.

Let $\mathrm{ff}^{o b}(G \times H), G, H \in \mathcal{F}_{1}(X)$, be the boundary face of $X_{o b}^{2}$ obtained by blowing up the component $G \times H$ of $(\partial X)^{2}$. Then $\mathrm{ff}^{o b}:=\bigcup_{G, H \in \mathcal{F}_{1}(X)} \mathrm{ff}^{o b}(G \times H)$ is called the overblown b-front face, and $\Delta_{o b}:={\overline{\left(\beta_{o b}^{2}\right)^{-1}\left(\Delta^{2} \backslash(\partial X)^{2}\right)}}^{X_{o b}^{2}}$ the lifted diagonal. The blow-down map $\beta_{o b}^{2}$ induces isomorphisms

$$
\begin{aligned}
\left(\beta_{o b}^{2}\right)^{*}: \dot{\mathcal{C}}^{\infty}\left(X^{2},{ }^{b} \Omega^{\frac{1}{2}}\right) & \longrightarrow \dot{\mathcal{C}}^{\infty}\left(X_{o b}^{2},{ }^{b} \Omega^{\frac{1}{2}}\right), \text { and } \\
\left(\beta_{o b}^{2}\right)_{*}: \mathcal{C}^{-\infty}\left(X_{o b}^{2},{ }^{b} \Omega^{\frac{1}{2}}\right) & \longrightarrow \mathcal{C}^{-\infty}\left(X^{2},{ }^{b} \Omega^{\frac{1}{2}}\right),
\end{aligned}
$$


and the lift of the Schwartz kernel $k_{a} \in \mathcal{C}^{-\infty}\left(X^{2},{ }^{b} \Omega^{\frac{1}{2}}\right)$ of a continuous, linear operator $a: \dot{\mathcal{C}}^{\infty}\left(X,{ }^{b} \Omega^{\frac{1}{2}}\right) \longrightarrow \mathcal{C}^{-\infty}\left(X,{ }^{b} \Omega^{\frac{1}{2}}\right)$ is still denoted by $\kappa_{a} \in \mathcal{C}^{-\infty}\left(X_{o b}^{2},{ }^{b} \Omega^{\frac{1}{2}}\right)$.

Definition 4.10. For $m \in \mathbb{R}$, we let $\Psi_{o b, c l}^{m}\left(X,{ }^{b} \Omega^{\frac{1}{2}}\right)$ be the space of all continuous linear operators $a: \dot{\mathcal{C}}^{\infty}\left(X,{ }^{b} \Omega^{\frac{1}{2}}\right) \longrightarrow \mathcal{C}^{-\infty}\left(X,{ }^{b} \Omega^{\frac{1}{2}}\right)$ whose lifted Schwartz kernels $\kappa_{a}$ belong to $I_{c l}^{m}\left(X_{o b}^{2}, \Delta_{o b} ; \Omega^{\frac{1}{2}}\right)$, and vanish with all derivatives at $\partial X_{o b}^{2} \backslash \mathrm{ff}^{o b}$. The elements of $\Psi_{o b, c l}^{m}\left(X,{ }^{b} \Omega^{\frac{1}{2}}\right)$ are called classical, overblown b-pseudodifferential operators of order $m$.

The space $\Psi_{o b, c l}^{m}\left(X,{ }^{b} \Omega^{\frac{1}{2}}\right)$ is slightly larger than $\Psi_{b, c l}^{m}\left(X,{ }^{b} \Omega^{\frac{1}{2}}\right)$ because the additional blow-up in the definition of $X_{o b}^{2}$ allows the overblown kernel to have nontrivial behavior also at the off-diagonal corners. This will be used to identify certain cone pseudodifferential operators in Section 5.1 that are not in the b-calculus. For an example of an element in $\Psi_{o b}^{-\infty}\left(X,{ }^{b} \Omega^{\frac{1}{2}}\right)$ that is not in $\Psi_{b}^{-\infty}\left(X,{ }^{b} \Omega^{\frac{1}{2}}\right)$, we refer to $[\mathbf{1 6}$, Example 5.2].

As for the b-calculus the homogeneous principal symbol for conormal distributions induces a map ${ }^{o b} \sigma^{(m)}: \Psi_{o b, c l}^{m}\left(X,{ }^{b} \Omega^{\frac{1}{2}}\right) \longrightarrow \mathcal{C}^{\infty}\left({ }^{b} S^{*} X\right)$. Since ${ }^{o b} \sigma^{(m)}(a)$ depends only on the behavior of $\kappa_{a}$ near $\Delta_{o b}$, we clearly have ${ }^{o b} \sigma^{(m)}(a)={ }^{b} \sigma^{(m)}(a)$ for all $a \in \Psi_{b, c l}^{m}\left(X,{ }^{b} \Omega^{\frac{1}{2}}\right) \subseteq \Psi_{o b, c l}^{m}\left(X,{ }^{b} \Omega^{\frac{1}{2}}\right)$. For the definition of the indicial family for the small overblown calculus, let $\varrho_{G}: X \longrightarrow \overline{\mathbb{R}}_{+}$be a defining function for $G \in \mathcal{F}_{1}(X), \varrho:=\prod_{G \in \mathcal{F}_{1}(X)} \varrho_{G}$, and $(\cdot)_{\partial}: \Psi_{o b, c l}^{m}\left(X,{ }^{b} \Omega^{\frac{1}{2}}\right) \longrightarrow \Psi_{c l}^{m}\left(\partial X, \Omega^{\frac{1}{2}}\right)$ be the morphism of restricting to the boundary. Exactly as in the b-case

$$
I_{\varrho}: \Psi_{o b, c l}^{m}\left(X,{ }^{b} \Omega^{\frac{1}{2}}\right) \longrightarrow M_{\mathcal{O}}^{m}\left(\partial X, \Omega^{\frac{1}{2}}\right): a \longmapsto\left[z \longmapsto\left(\varrho^{z} a \varrho^{-z}\right)_{\partial}\right]
$$

is said to be the indicial family of $a$. For $a \in \Psi_{b, c l}^{m}\left(X,{ }^{b} \Omega^{\frac{1}{2}}\right) \subseteq \Psi_{o b, c l}^{m}\left(X,{ }^{b} \Omega^{\frac{1}{2}}\right)$ we have $I_{\varrho}(a)=\operatorname{diag}\left(I_{\varrho G}^{(G)}(a): G \in \mathcal{F}_{1}(X)\right)$. With almost the same proof as for the b-calculus we can characterize those overblown b-pseudodifferential operators that are Fredholm. For simplicity, let $\mathfrak{b} \in \mathbb{R}$ be the multi-weight $\mathfrak{b}_{G}=\mathfrak{b}$.

Theorem 4.11. Let $a \in \Psi_{o b, c l}^{m}\left(X,{ }^{b} \Omega^{\frac{1}{2}}\right)$ and $\mathfrak{b}, s \in \mathbb{R}$ be arbitrary. Then $a$ extends to a bounded operator $a: \varrho^{\mathfrak{b}} H_{b}^{s}\left(X,{ }^{b} \Omega^{\frac{1}{2}}\right) \longrightarrow \varrho^{\mathfrak{b}} H_{b}^{s-m}\left(X,{ }^{b} \Omega^{\frac{1}{2}}\right)$ which is Fredholm if and only if ${ }^{o b} \sigma^{(m)}(a)(\zeta) \neq 0$ for all $\zeta \in{ }^{b} S^{*} X$, and, for all $\lambda \in \mathbb{R}$, the operator $I_{\varrho}(a)(i \lambda-\mathfrak{b}): H^{s}\left(\partial X, \Omega^{\frac{1}{2}}\right) \longrightarrow H^{s-m}\left(\partial X, \Omega^{\frac{1}{2}}\right)$ is invertible.

Note that for $a \in \Psi_{b, c l}^{m}\left(X,{ }^{b} \Omega^{\frac{1}{2}}\right) \subseteq \Psi_{o b, c l}^{m}\left(X,{ }^{b} \Omega^{\frac{1}{2}}\right)$ Theorem 4.11 specializes to Theorem 4.8 .

\section{Comparison of b-calculus and cone algebra}

5.1. The small calculus and the holomorphic cone algebra. As in Subsection 3.2 , let $(x, y): X \supseteq U \stackrel{\cong}{\longrightarrow} \overline{\mathbb{R}}_{+} \times \partial X$ be a collar neighborhood of the boundary, and choose a defining function $\varrho: X \longrightarrow \overline{\mathbb{R}}_{+}$of $\partial X$ with $\left.\varrho\right|_{U}=x$. Without loss of generality we can assume $\varrho=\prod_{H \in \mathcal{F}_{1}(X)} \varrho_{H}$ where $\varrho_{H}: X \longrightarrow \overline{\mathbb{R}}_{+}$ is a defining function for the component $H \in \mathcal{F}_{1}(X)$ of $\partial X$. 
Proposition 5.1. For $\gamma, m \in \mathbb{R}$, we have

$$
\Psi_{b, c l}^{m}\left(X,{ }^{b} \Omega^{\frac{1}{2}}\right) \subseteq \Psi_{o b, c l}^{m}\left(X,{ }^{b} \Omega^{\frac{1}{2}}\right) \subseteq \varrho^{m} \mathbb{C}^{m}\left(X,{ }^{b} \Omega^{\frac{1}{2}} ;(\gamma, \gamma-m, \infty)\right)_{O},
$$

${ }^{b} \sigma^{(m)}(a)={ }^{b} \sigma_{\psi, \mathfrak{C}}^{(m)}\left(\varrho^{-m} a\right)$ and $I_{a}=\sigma_{M}^{(m)}\left(\varrho^{-m} a\right) \in M_{\mathcal{O}}^{m}\left(\partial X, \Omega^{\frac{1}{2}}\right)$ for all operators $a \in \Psi_{o b, c l}^{m}\left(X,{ }^{b} \Omega^{\frac{1}{2}}\right)$.

Proof. An inspection of the proof of [16, Proposition 5.4] shows that the Proposition holds for $a \in \Psi_{b, c l}^{m}\left(X,{ }^{b} \Omega^{\frac{1}{2}}\right)$. If the kernel $\kappa_{a}$ of $a \in \Psi_{o b, c l}^{m}\left(X,{ }^{b} \Omega^{\frac{1}{2}}\right)$ is supported away from the faces $\mathrm{ff}^{o b}(G \times H)$ for all $G, H \in \mathcal{F}_{1}(X)$ with $G \neq H$, then we have $a \in \Psi_{b, c l}^{m}\left(X,{ }^{b} \Omega^{\frac{1}{2}}\right)$; thus, we can assume that $\kappa_{a}$ is supported in a coordinate patch near $\mathrm{ff}^{o b}(G \times H), G \neq H$, and we have $\kappa_{a}=\widehat{\kappa}_{a}\left(s, x, y, y^{\prime}\right)\left|\frac{d s}{s} \frac{d x}{x} d y d y^{\prime}\right|^{\frac{1}{2}}$ with $\widehat{\kappa}_{a} \in \dot{\mathcal{S}}\left(\overline{\mathbb{R}}_{+}\right) \widehat{\otimes}_{\pi} \mathcal{C}_{c}^{\infty}\left(\overline{\mathbb{R}}_{+}\right) \widehat{\otimes}_{\pi} \mathcal{C}_{c}^{\infty}\left(\mathbb{R}_{y}^{n-1}\right) \widehat{\otimes}_{\pi} \mathcal{C}_{c}^{\infty}\left(\mathbb{R}_{y^{\prime}}^{n-1}\right)$ by Definition 4.10 , where we have used the projective coordinates $s=\frac{x}{x^{\prime}}, x, y(\in G), y^{\prime}(\in H)$ near $\mathrm{ff}^{o b}(G \times H)$ and $\dot{\mathcal{S}}$ stands for the space of smooth functions $\overline{\mathbb{R}}_{+} \longrightarrow \mathbb{C}$ that vanish with all derivatives at $s=0$ and rapidly for $s \rightarrow \infty$. Mellin transform with respect to the variable $s$ yields

$$
h_{a}=M_{s \rightarrow z} \kappa_{a} \in \mathcal{C}_{c}^{\infty}\left(\overline{\mathbb{R}}_{+}, M_{\mathcal{O}}^{-\infty}\left(G, H, \Omega^{\frac{1}{2}}\right)\right) \subseteq \mathcal{C}_{c}^{\infty}\left(\overline{\mathbb{R}}_{+}, M_{\mathcal{O}}^{-\infty}\left(\partial X, \Omega^{\frac{1}{2}}\right)\right),
$$

Then the Schwartz kernel $k_{\varrho^{-m} a}=\widehat{k}_{\varrho^{-m} a}\left(x, y, x^{\prime}, y^{\prime}\right)\left|\frac{d x}{x} d y \frac{d x^{\prime}}{x^{\prime}} d y^{\prime}\right|^{\frac{1}{2}}$ of $\varrho^{-m} a$ is supported near $x, x^{\prime}=0$, and satisfies

$$
\widehat{k}_{\varrho^{-m} a}\left(x, y, x^{\prime}, y^{\prime}\right)=x^{-m} \int_{\mathbb{R}_{\xi}}\left(\frac{x}{x^{\prime}}\right)^{-(\mu+i \xi)} \widehat{h}_{a}(x, \mu+i \xi)\left(y, y^{\prime}\right) d \xi
$$

for all $\mu \in \mathbb{R}$ by the analyticity of $h_{a}$, i.e. for appropriate cut-off functions $\omega, \omega_{1}$

$$
\varrho^{-m} a=\omega x^{-m} \operatorname{op}_{M}^{\left(\gamma-\frac{n-1}{2}\right)}(h) \omega_{1} \in \mathfrak{C}^{m}\left(X,{ }^{b} \Omega^{\frac{1}{2}} ;(\gamma, \gamma-m, \infty)\right)_{O}
$$

with ${ }^{b} \sigma^{(m)}(a)={ }^{b} \sigma_{\psi, \mathcal{C}}^{(m)}\left(\varrho^{-M} a\right)=0$ and by the definition of the indicial family and the conormal symbol we have $I_{a}=h_{a}(0)=\sigma_{M}^{(m)}\left(\varrho^{-m} a\right) \in M_{\mathcal{O}}^{-\infty}\left(G, H, \Omega^{\frac{1}{2}}\right)$. This completes the proof.

We consider now the other inclusion, and identify certain parts of the cone algebra with elements in the overblown b-calculus. By the very definition of the latter we certainly have

$$
(1-\omega) a_{\psi}\left(1-\omega_{1}\right) \in \Psi_{b, c l}^{m}\left(X,{ }^{b} \Omega^{\frac{1}{2}}\right) \subseteq \Psi_{o b, c l}^{m}\left(X,{ }^{b} \Omega^{\frac{1}{2}}\right)
$$

for all $a_{\psi} \in \Psi_{c l}^{m}\left(X_{0}, \Omega^{\frac{1}{2}}\right)$ and all cut-off functions $\omega, \omega_{1} \in \mathcal{C}_{c}^{\infty}\left(\overline{\mathbb{R}}_{+}\right)$.

Lemma 5.2. For $\omega, \omega_{0} \in \mathcal{C}_{c}^{\infty}\left(\overline{\mathbb{R}}_{+}\right), h \in \mathcal{C}_{c}^{\infty}\left(\overline{\mathbb{R}}_{+}, M_{\mathcal{O}}^{m}\left(\partial X, \Omega^{\frac{1}{2}}\right)\right)$, and any $\mu \in \mathbb{R}$ we have

$$
\varrho^{m} \omega x^{-m} \operatorname{op}_{M}^{\left(\frac{1}{2}-\mu\right)}(h) \omega_{0} \in \Psi_{o b, c l}^{m}\left(X,{ }^{b} \Omega^{\frac{1}{2}}\right)
$$


Proof. Let $\partial X=\biguplus_{j=1}^{P} H_{j}$ be the decomposition of $\partial X$ into connected components, and $\left(h_{j, k}\right)_{j, k=1, \ldots, P}$ be the corresponding decomposition for $h$. In [16, Proposition 5.1] we have shown

$$
\varrho^{m} \omega x^{-m} \operatorname{op}_{M}^{\left(\frac{1}{2}-\mu\right)}\left(h_{j, j}\right) \omega_{0} \in \Psi_{b, c l}^{m}\left(X,{ }^{b} \Omega^{\frac{1}{2}}\right) \subseteq \Psi_{o b, c l}^{m}\left(X,{ }^{b} \Omega^{\frac{1}{2}}\right),
$$

therefore, it remains to consider $h_{j, k} \in \mathcal{C}^{\infty}\left(\overline{\mathbb{R}}_{+}, M_{\mathcal{O}}^{-\infty}\left(H_{j}, H_{k}, \Omega^{\frac{1}{2}}\right)\right)$ for $j \neq k$. By a partition of unity we can assume $h_{j, k} \in \mathcal{C}_{c}^{\infty}\left(\overline{\mathbb{R}}_{+}, \mathcal{O}\left(\mathbb{C}, \mathcal{C}^{\infty}\left(\mathbb{R}_{y}^{n-1} \times \mathbb{R}_{y^{\prime}}^{n-1}\right)\right)\right)$ such that $h_{j, k}\left(x, y, y^{\prime}, \gamma+i \xi\right) \in \mathcal{C}^{\infty}\left(\overline{\mathbb{R}}_{+}, \mathcal{S}\left(\mathbb{R}_{\xi}, \mathcal{C}^{\infty}\left(\mathbb{R}_{y}^{n-1} \times \mathbb{R}_{y^{\prime}}^{n-1}\right)\right)\right)$ holds uniformly for $|\gamma| \leq R$, and $\operatorname{supp} h_{j, k}(x, \cdot, \cdot, z) \subseteq L$ for all $x \in \overline{\mathbb{R}}_{+}$, all $z \in \mathbb{C}$, and a fixed compact set $L \subseteq \mathbb{R}_{y}^{n-1} \times \mathbb{R}_{y^{\prime}}^{n-1}$. We use the singular coordinates $\tau=\frac{x-x^{\prime}}{x+x^{\prime}}$ and $r=x+x^{\prime}$ near $\mathrm{ff}^{o b}\left(H_{j} \times H_{k}\right)$. The Schwartz kernel of $a=\omega \mathrm{op}_{M}^{\left(\frac{1}{2}-\mu\right)}\left(h_{j, k}\right) \omega_{0}$ is given by

$$
\begin{aligned}
k_{a} & =\hat{k}_{a}\left(x, y, x^{\prime}, y^{\prime}\right)\left|\frac{d x}{x} d y \frac{d x^{\prime}}{x^{\prime}} d y^{\prime}\right|^{\frac{1}{2}} \\
& =\omega(x) \omega_{0}\left(x^{\prime}\right) \int_{\mathbb{R}_{\xi}}\left(\frac{x}{x^{\prime}}\right)^{-(\mu+i \xi)} \widehat{h}_{j, k}\left(x, y, y^{\prime}, \mu+i \xi\right) d \xi\left|\frac{d x}{x} d y \frac{d x^{\prime}}{x^{\prime}} d y^{\prime}\right|^{\frac{1}{2}},
\end{aligned}
$$

thus, the lift to $X_{o b}^{2}$ has the form $\kappa_{a}=\widehat{\kappa}_{a}\left(\tau, r, y, y^{\prime}\right)\left|\frac{d \tau}{1-\tau^{2}} \frac{d r}{r} d y d y^{\prime}\right|^{\frac{1}{2}}$ with

$$
\begin{aligned}
\widehat{\kappa}_{a}\left(\tau, r, y, y^{\prime}\right)=\omega\left(\frac{r}{2}(1+\tau)\right) \omega_{0}\left(\frac{r}{2}(1-\tau)\right)\left(\frac{1+\tau}{1-\tau}\right)^{-\mu} \\
\int_{\mathbb{R}_{\xi}}\left(\frac{1+\tau}{1-\tau}\right)^{i \xi} \widehat{h}_{j, k}\left(\frac{r}{2}(1+\tau), y, y^{\prime}, \mu+i \xi\right) d \xi .
\end{aligned}
$$

By [16, Lemma 2.1] we know that $\kappa_{a} \in \mathcal{C}^{\infty}\left((-1,1) \times \overline{\mathbb{R}}_{+} \times \mathbb{R}_{y}^{n-1} \times \mathbb{R}_{y^{\prime}}^{n-1},{ }^{b} \Omega^{\frac{1}{2}}\right)$ vanishes with all derivatives as $\tau \rightarrow \pm 1$, i.e. $a \in \Psi_{o b, c l}^{m}\left(X,{ }^{b} \Omega^{\frac{1}{2}}\right)$.

REMARK 5.3. If the boundary $\partial X$ is not connected, then we do not have $\omega \operatorname{op}_{M}^{\left(\frac{1}{2}-\mu\right)}(h) \omega_{0} \in \Psi_{b, c l}^{m}\left(X,{ }^{b} \Omega^{\frac{1}{2}}\right)$ in general, because the kernels corresponding to the off-diagonal elements of $h$ do not vanish with all derivatives at $H_{j} \times H_{k} \subseteq X_{b}^{2}$. For a concrete example see [16, Example 5.2]. The overblown b-double space $X_{o b}^{2}$ resolves this singularity by blowing up additionally the corners $H_{j} \times H_{k}$.

Theorem 5.4. For $\gamma, m \in \mathbb{R}$ and $j \in \mathbb{N}_{0}$, we have

$$
\mathfrak{C}^{m-j}\left(X,{ }^{b} \Omega^{\frac{1}{2}} ;(\gamma, \gamma-m, \infty)\right)_{O}=\varrho^{j-m} \Psi_{o b, c l}^{m-j}\left(X,{ }^{b} \Omega^{\frac{1}{2}}\right) .
$$

Proof. By Proposition 5.1, the left-hand side contains the right-hand side, whereas a combination of (5.2), Lemma 5.2, and the remark after Definition 3.9 yields the other inclusion. Recall that the left hand side is, in fact, independent of the weigth data $(\gamma, \gamma-m)$. 
5.2. Green operators and $\Psi^{-\infty, \mathcal{E}}\left(X,{ }^{b} \Omega^{\frac{1}{2}}\right)$. To compare the residual operators of the cone algebra and the $b$-calculus, let us introduce $\mathcal{C}_{\gamma}^{k}(X, F), k \in \mathbb{N}_{0} \cup\{\infty\}$, $\gamma \in \mathbb{R}, F$ a Fréchet space, as the space of all functions $f \in \mathcal{C}^{k}\left(X_{0}, F\right)$ such that

$$
\operatorname{Diff}_{b}^{k}(X) f \in \varrho^{\gamma-\frac{n}{2}}(\log \varrho)^{-l} L^{\infty}\left(X_{0}, F\right) \quad \forall l \leq k .
$$

This is a Fréchet space in a natural way, which does not depend on the choice of the boundary defining function $\varrho$. If $F=\mathbb{C}$, we omit it from the notation. It is then straightforward to show:

LEMma 5.5. For any $k \in \mathbb{N}_{0} \cup\{\infty\}$

$$
\bigcup_{\varepsilon>0} \mathcal{C}_{\frac{n}{2}+k+\varepsilon}^{k}(X, F) \subset \dot{\mathcal{C}}^{k}(X, F) \subset \bigcap_{\varepsilon>0} \mathcal{C}_{\frac{n}{2}+k-\varepsilon}^{k}(X, F) .
$$

Recall that $\dot{\mathcal{C}}^{k}(X, F)$ denotes all k-times differentiable functions that vanish up to order $k$ at the boundary.

Corollary 5.6. Let $X$ be a manifold with connected boundary. If $E$ is an index set for $X, \gamma<\frac{n}{2}+\inf E$ and

$$
Q=\left\{(-z, k):(z, k) \in E \text { and }\left(z, k^{\prime}\right) \in E \Rightarrow k^{\prime} \leq k\right\}
$$

then

$$
\mathcal{A}_{p h g}^{E}\left(X,{ }^{b} \Omega^{\frac{1}{2}}\right)=\mathcal{C}_{\gamma, Q, w}^{\infty}\left(X,{ }^{b} \Omega^{\frac{1}{2}}\right)
$$

Let us recall that the elements of the completed $\pi$-tensor product $F^{0} \hat{\otimes}_{\pi} F^{1}$ of Fréchet spaces $F^{0}, F^{1}$ are precisely of the form $\sum_{j=0}^{\infty} \lambda_{j} f_{j}^{(0)} \otimes f_{j}^{(1)}$ with an absolutely summable scalar sequence $\left(\lambda_{j}\right)_{j}$, and sequences $\left(f_{j}^{(l)}\right)_{j}$ converging to 0 in $F^{l}$. To have at hand a short notion, let us refer to this as a 'projective sum'.

Proposition 5.7. Let $\mathcal{E}$ and $\mathcal{F}$ be index families for $X$. Then

$$
\mathcal{A}_{p h g}^{(\mathcal{E}, \mathcal{F})}\left(X^{2}\right)=\mathcal{A}_{p h g}^{\mathcal{E}}(X) \hat{\otimes}_{\pi} \mathcal{A}_{p h g}^{\mathcal{F}}(X) .
$$

Here, the pure tensors are defined by $(u \otimes v)\left(x, x^{\prime}\right)=u(x) v\left(x^{\prime}\right), x, x^{\prime} \in X$.

Proof. For a Fréchet space $F$, we let $\mathcal{A}_{p h g}^{\mathcal{E}}(X ; F)$ denote the space of all functions $u$ smooth in the interior of $X$ with values in $F$, such that for any (connected) boundary component $H \in \mathcal{F}_{1}(X)$ and all $N \in \mathbb{N}$ we can write $\varphi_{H} u=u_{N}^{(H)}+r_{N}^{(H)}$ with

$$
u_{N}^{(H)}=\sum_{(z, k) \in E_{H}, \operatorname{Re} z \leq N} \varrho_{H}^{z}\left(\log \varrho_{H}\right)^{k} a_{z, k}^{(H)}, \text { and } r_{N}^{(H)} \in \dot{\mathcal{C}}^{N}(X, F)
$$

for appropriate $a_{z, k}^{(H)} \in \mathcal{C}^{\infty}(X, F)$. Here $\left(\varphi_{H}\right)_{H}$ is a partition of unity on $X$ with $H \subset \operatorname{supp} \varphi_{H}$ and $G \cap \operatorname{supp} \varphi_{H}=\emptyset$ for all boundary surfaces $G \neq H$. The topology is induced by the coefficients $a_{z, k}^{(H)}$ and the remainders $r_{N}^{(H)}$ in a straightforward way. Since $\mathcal{A}_{p h g}^{(0, \mathcal{F})}\left(X^{2}\right)=\mathcal{C}^{\infty}\left(X, \mathcal{A}_{p h g}^{\mathcal{F}}(X)\right)$ it is obvious from Definition 4.4 , that

$$
\mathcal{A}_{p h g}^{(\mathcal{E}, \mathcal{F})}\left(X^{2}\right)=\mathcal{A}_{p h g}^{\mathcal{E}}\left(X ; \mathcal{A}_{p h g}^{\mathcal{F}}(X)\right)
$$


Note that in (4.5) the remainder belongs to $\dot{\mathcal{C}}_{H}^{N}$ rather than $\dot{\mathcal{C}}^{N}$. However, this makes no difference, since $\varphi_{H} u$ is located away from all boundary surfaces different from $H$. To verify the proposition, it is therefore sufficient to show that $\mathcal{A}_{p h g}^{\mathcal{E}}(X ; F)=\mathcal{A}_{p h g}^{\mathcal{E}}(X) \widehat{\widehat{\Theta}}_{\pi} F$.

Step 1: Let $u \in \mathcal{A}_{p h g}^{\mathcal{E}}(X) \widehat{\otimes}_{\pi} F$ have a representation $\sum_{j} \lambda_{j} u_{j} f_{j}$ as a projective sum with $u_{j} \in \mathcal{A}_{p h g}^{\mathcal{E}}(X)$ and $f_{j} \in F$. Inserting $\varphi_{H} u_{j}=u_{j, N}^{(H)}+r_{j, N}^{(H)}$ as above, we obtain

$$
\varphi_{H} u=\sum_{(z, k) \in E_{H}, \operatorname{Re} z \leq N} \varrho_{H}^{z}\left(\log \varrho_{H}\right)^{k}\left(\sum_{j} \lambda_{j} a_{j, z, k}^{(H)} f_{j}\right)+\sum_{j} \lambda_{j} r_{j, N}^{(H)} f_{j}
$$

with $a_{j, z, k}^{(H)} \in \mathcal{C}^{\infty}(X)$. This shows $u \in \mathcal{A}_{p h g}^{\mathcal{E}}(X, F)$, since $\mathcal{C}^{\infty}(X) \hat{\otimes}_{\pi} F=\mathcal{C}^{\infty}(X, F)$ and $\dot{\mathcal{C}}^{N}(X) \hat{\otimes}_{\pi} F \subset \dot{\mathcal{C}}^{N}(X, F)$.

Step 2a: Let $u \in \mathcal{A}_{p h g}^{\mathcal{E}}(X ; F)$ and $\gamma \in \mathbb{R}$ be with $\gamma<\frac{n}{2}+\inf E_{H}$ for all boundary surfaces $H$ of $X$. Write $u=\sum_{H} u_{N}^{(H)}$ modulo $\dot{\mathcal{C}}^{N}(X, F)$. We may assume that each $u_{N}^{(H)}$ is supported away from any boundary surface different from $H$. Therefore, $u_{N}^{(H)} \in \mathcal{C}_{\gamma}^{\infty}(X, F)$. Moreover, for any given $k \in \mathbb{N}$ we have $\dot{\mathcal{C}}^{N}(X, F) \subset$ $\mathcal{C}_{\gamma}^{k}(X, F)$ for all sufficiently large $N$ by Lemma 5.5. This yields

$$
u \in \bigcap_{k \in \mathbb{N}} C_{\gamma}^{k}(X, F)=\mathcal{C}_{\gamma}^{\infty}(X, F)=\mathcal{C}_{\gamma}^{\infty}(X) \widehat{\otimes}_{\pi} F
$$

The last identity follows from $\mathcal{C}^{\infty}(X, F)=\mathcal{C}^{\infty}(X) \hat{\otimes}_{\pi} F$ and - via local coordinates near the boundary - from $\mathcal{S}\left(\mathbb{R}^{n}, F\right)=\mathcal{S}\left(\mathbb{R}^{n}\right) \widehat{\otimes}_{\pi} F$, where $\mathcal{S}$ denotes the space of rapidly decreasing functions.

Step 2b: Let $u \in \mathcal{A}_{p h g}^{\mathcal{E}}(X ; F)$. Inserting in the above decomposition of $u$ for $a_{z, k} \in \mathcal{C}^{\infty}(X, F)=\mathcal{C}^{\infty}(X) \widehat{\otimes}_{\pi} F$ a projective sum, we get $u_{N}^{(H)} \in \mathcal{A}_{p h g}^{\mathcal{E}}(X) \widehat{\otimes}_{\pi} F$ (without loss of generality, $u_{N}^{(H)}$ is supported away from any boundary component different from $H$ ). Then, using Step 2a,

$$
r_{N}:=\sum_{H \in \mathcal{F}_{1}(X)}\left(\varphi_{H} u-u_{N}^{(H)}\right) \in \mathcal{A}_{p h g}^{\mathcal{E}^{(N)}}(X ; F) \subset \mathcal{C}_{\gamma+N}^{\infty}(X) \hat{\otimes}_{\pi} F
$$

where $\mathcal{E}^{(N)}$ is defined by $E_{H}^{(N)}=\left\{(z, k) \in E_{H}: \operatorname{Re} z>N\right\}$. Thus, for any $N$,

$$
u=\sum_{H \in \mathcal{F}_{1}(X)} \varphi_{H} u=\sum_{H \in \mathcal{F}_{1}(X)} u_{N}^{(H)}+r_{N} \in\left(\mathcal{A}_{p h g}^{\mathcal{E}}(X)+\mathcal{C}_{\gamma+N}^{\infty}(X)\right) \widehat{\otimes}_{\pi} F
$$

Since $\cap_{N}\left(\mathcal{A}_{p h g}^{\mathcal{E}}(X)+\mathcal{C}_{\gamma+N}^{\infty}(X)\right)=\mathcal{A}_{p h g}^{\mathcal{E}}(X)$ by Lemma 5.5 , this, finally, yields $u \in \mathcal{A}_{p h g}^{\mathcal{E}}(X) \hat{\otimes}_{\pi} F$.

Theorem 5.8. Let the boundary of $X$ be connected, $\left(E_{0}, E_{1}\right)$ be an index set for $X^{2}$, where $E_{0}$ corresponds to the left boundary $\partial X \times X$ and $E_{1}$ to the right boundary $X \times \partial X$. For $j=0,1$, let $\gamma_{j}<(-1)^{j} \frac{n}{2}+\inf E_{j}$ and $Q_{j} \in \operatorname{As}_{w}\left(\gamma_{j}, \infty\right)$ be 
given by

$$
Q_{j}=\left\{(-z+j n, k):(z, k) \in E_{j} \text { and }\left(z, k^{\prime}\right) \in E_{j} \Rightarrow k^{\prime} \leq k\right\} .
$$

Then

$$
\Psi^{-\infty,\left(E_{0}, E_{1}\right)}\left(X,{ }^{b} \Omega^{\frac{1}{2}}\right)=\mathfrak{C}_{G, w}\left(X,{ }^{b} \Omega^{\frac{1}{2}},\left(-\gamma_{1}, \gamma_{0}, \infty\right)\right)_{Q_{0}, \bar{Q}_{1}} .
$$

Proof. By definition, the elements of $\Psi^{-\infty,\left(E_{0}, E_{1}\right)}\left(X,{ }^{b} \Omega^{\frac{1}{2}}\right)$ are precisely the integral operators with respect to the $L^{2}\left(X,{ }^{b} \Omega^{\frac{1}{2}}\right)$-scalar product and kernel in $\mathcal{A}_{p h g}^{\left(E_{0}, E_{1}\right)}\left(X^{2},{ }^{b} \Omega^{\frac{1}{2}}\right)$, i.e. $a u(x)=\left\langle k_{a}(x, \cdot), \bar{u}\right\rangle_{L^{2}}$.

Now, an operator has an integral kernel $\kappa$ with respect to the scalar product in $L^{2}\left(X,{ }^{b} \Omega^{\frac{1}{2}}\right)$ if and only if it has kernel $\tilde{\kappa}\left(x, x^{\prime}\right)=\varrho\left(x^{\prime}\right)^{-n} \kappa\left(x, x^{\prime}\right)$ with respect to the scalar product in $\mathcal{H}^{0,0}\left(X,{ }^{b} \Omega^{\frac{1}{2}}\right)=\varrho^{-\frac{n}{2}} L^{2}\left(X,{ }^{b} \Omega^{\frac{1}{2}}\right)$.

The result thus follows from Proposition 5.7, Corollary 5.6, and

$$
\varrho^{-n} C_{\gamma, Q, w}^{\infty}\left(X,{ }^{b} \Omega^{\frac{1}{2}}\right)=C_{\gamma-n, Q(n), w}^{\infty}\left(X,{ }^{b} \Omega^{\frac{1}{2}}\right)
$$

with $Q(n)=\left\{\left(q+n, l_{q}\right):\left(q, l_{q}\right) \in Q\right\}$.

Using the simple fact that $\varrho^{\sigma} \mathfrak{C}_{G, w}\left(X,{ }^{b} \Omega^{\frac{1}{2}},\left(\gamma, \gamma^{\prime}, k\right)\right)=\mathfrak{C}_{G, w}\left(X,{ }^{b} \Omega^{\frac{1}{2}},\left(\gamma, \gamma^{\prime}+\sigma, k\right)\right)$, it is immediate from the latter theorem, that

$$
\mathfrak{C}_{G, w}\left(X,{ }^{b} \Omega^{\frac{1}{2}},(\gamma, \gamma-m, \infty)\right)=\bigcup_{\mathcal{E}} \varrho^{-m+j} \Psi^{-\infty, \mathcal{E}}\left(X,{ }^{b} \Omega^{\frac{1}{2}}\right)
$$

with the union taken over all index families $\mathcal{E}=\left(E_{0}, E_{1}\right)$ with

$$
-\inf E_{1}<\gamma-\frac{n}{2}<\inf E_{0}+j .
$$

5.3. The kernel characterization for weak Green operators. In this section we shall give the proof of the kernel characterization for weak Green operators, cf. the end of Subsection 3.3. It relies on the following Proposition 5.9, which can be found for instance in [22, Theorem 2.1] or [35, Proposition 4.14.2]. convenience, we state this result in the terminology used in Section 3. We set

$\mathcal{C}_{\gamma, \gamma^{\prime}}^{\infty}\left(X^{2}\right)=\mathcal{C}_{\gamma}^{\infty}\left(X, \mathcal{C}_{\gamma^{\prime}}^{\infty}(X)\right)=\mathcal{C}_{\gamma}^{\infty}(X) \hat{\otimes}_{\pi} \mathcal{C}_{\gamma^{\prime}}^{\infty}(X), \quad \mathcal{C}_{-\infty,-\infty}^{\infty}\left(X^{2}\right)=\bigcup_{\gamma \in \mathbb{R}} \mathcal{C}_{\gamma, \gamma}^{\infty}\left(X^{2}\right)$

and similarly for sections into ${ }^{b} \Omega^{\frac{1}{2}}\left(X^{2}\right)$. In fact, the latter space coicides with $\mathcal{A}\left(X^{2}\right)$ introduced in $(2.1)$.

Proposition 5.9. Let $Q_{j} \in \operatorname{As}_{w}\left(\gamma_{j}, \infty\right)$ and $a_{N}^{j}=a_{N}\left(Q_{j}\right), N \in \mathbb{N}$, a sequence of differential operators acting on b-half densities on $X$ such that, in local coordinates near the boundary,

$$
a_{N}^{j}=\prod_{\left(q, l_{q}\right) \in Q_{j}, \operatorname{Req} \geq \frac{n}{2}-\gamma_{j}-N}\left(x \partial_{x}+q\right)^{l_{q}} .
$$

For $u \in \mathcal{C}_{-\infty,-\infty}^{\infty}\left(X^{2},{ }^{b} \Omega^{\frac{1}{2}}\right)$, we have $u \in \mathcal{C}_{\gamma_{0}, Q_{0}, w}^{\infty}\left(X,{ }^{b} \Omega^{\frac{1}{2}}\right) \widehat{\otimes}_{\pi} \mathcal{C}_{\gamma_{1}, Q_{1}, w}^{\infty}\left(X,{ }^{b} \Omega^{\frac{1}{2}}\right)$ if and only if

$$
a_{N_{0}}^{0} a_{N_{1}}^{1} u \in \mathcal{C}_{\gamma_{0}+N_{0}, \gamma_{1}+N_{1}}^{\infty}\left(X^{2},{ }^{b} \Omega^{\frac{1}{2}}\right) \quad \forall N_{0}, N_{1} \in \mathbb{N},
$$


where $a_{N_{0}}^{0}$ acts on the first variable, and $a_{N_{1}}^{1}$ on the second one. An analogous result holds for functions of only one variable.

Now let $G \in \mathfrak{C}_{G, w}\left(X,{ }^{b} \Omega^{\frac{1}{2}},\left(\gamma_{0}, \gamma_{1}, \infty\right)\right)_{Q_{1}, Q_{0}}$ be a weak Green operator. We shall show that $G u(x)=\langle k(x, \cdot), \bar{u}\rangle_{0,0}$ with

$$
k \in \mathcal{C}_{\gamma_{1}, Q_{1}, w}^{\infty}\left(X,{ }^{b} \Omega^{\frac{1}{2}}\right) \widehat{\otimes}_{\pi} \mathcal{C}_{-\gamma_{0}, \bar{Q}_{0}}^{\infty}\left(X,{ }^{b} \Omega^{\frac{1}{2}}\right) .
$$

A first observation is that $G$ has a kernel $k \in \mathcal{C}_{\gamma_{1},-\gamma_{0}}^{\infty}\left(X^{2},{ }^{b} \Omega^{\frac{1}{2}}\right)$ (this can be deduced from the fact that an operator $A \in \mathcal{L}\left(L^{2}\left(\mathbb{R}^{n}\right)\right)$ such that $A, A^{*}$ : $L^{2}\left(\mathbb{R}^{n}\right) \rightarrow \mathcal{S}\left(\mathbb{R}^{n}\right)$ has a kernel in $\left.\mathcal{S}\left(\mathbb{R}^{2 n}\right)\right)$. Set $G\left(N_{0}, N_{1}\right)=a_{N_{1}}^{1} G\left(a_{N_{0}}^{0}\right)^{*}$, where $a_{N_{j}}^{j}=a_{N_{j}}\left(Q_{j}\right)$ is a sequence of differential operators as in (5.7) and * denotes the adjoint with respect to the $\mathcal{H}^{0,0}\left(X,{ }^{b} \Omega^{\frac{1}{2}}\right)$-scalar product. By the latter proposition and again the above argument, it follows that $G\left(N_{0}, N_{1}\right)$ has a kernel

$$
k\left(N_{0}, N_{1}\right) \in \mathcal{C}_{\gamma_{1}+N_{1},-\gamma_{0}+N_{0}}^{\infty}\left(X^{2},{ }^{b} \Omega^{\frac{1}{2}}\right) .
$$

But now

$$
\begin{aligned}
\left\langle k\left(N_{0}, N_{1}\right)(x, \cdot), \bar{u}\right\rangle_{0,0} & =G\left(N_{0}, N_{1}\right) u(x)=a_{N_{1}}^{1}\left\langle k(x, \cdot), \overline{\left(a_{N_{0}}^{0}\right)^{*} u}\right\rangle_{0,0} \\
& =\left\langle a_{N_{1}}^{1} k(x, \cdot), a_{N_{0}}\left(\bar{Q}_{0}\right)^{*} \bar{u}\right\rangle_{0,0}=\left\langle a_{N_{1}}^{1} a_{N_{0}}\left(\bar{Q}_{0}\right) k(x, \cdot), \bar{u}\right\rangle_{0,0}
\end{aligned}
$$

shows that $a_{N_{1}}\left(Q_{1}\right) a_{N_{0}}\left(\bar{Q}_{0}\right) k=k\left(N_{0}, N_{1}\right)$. Since this is true for all $N_{0}, N_{1}$, Proposition 5.9 yields the desired property of $k$.

5.4. Smoothing Mellin operators and $\widetilde{\Psi}_{b}^{-\infty, \mathcal{E}}\left(X,{ }^{b} \Omega^{\frac{1}{2}}\right)$. Throughout this subsection the boundary $\partial X$ of $X$ is supposed to be connected. Let us first show that smoothing Mellin operators belong to the spaces $\widetilde{\Psi}_{b}^{-\infty, \mathcal{E}}\left(X,{ }^{b} \Omega^{\frac{1}{2}}\right)$. Indeed, for $\mu, m \in \mathbb{R}, \ell_{0} \in \mathbb{N}_{0}$ and a weak asymptotic type $P=\left\{\left(p, n_{p}\right): p \in \pi_{\mathbb{C}} P\right\}$ satisfying $\pi_{\mathbb{C}} P \cap \Gamma_{\mu}=\emptyset$, let the index family $\mathcal{E}=\mathcal{E}\left(P, \mu, \ell_{0}\right)$ for $X_{b}^{2}$ be given by

$$
\begin{aligned}
\mathcal{E}(\mathrm{lb}) & :=\left\{\left(-p+\ell_{0}+i, k\right) \in \mathbb{C} \times \mathbb{N}_{0}: \operatorname{Re}(p)<\mu, i \in \mathbb{N}_{0}, 0 \leq k \leq n_{p}\right\}, \\
\mathcal{E}(\mathrm{rb}) & :=\left\{(p+i, k) \in \mathbb{C} \times \mathbb{N}_{0}: \operatorname{Re}(p)>\mu, i \in \mathbb{N}_{0}, 0 \leq k \leq n_{p}\right\}, \text { and } \\
\mathcal{E}\left(\mathrm{ff}^{b}\right) & :=0 .
\end{aligned}
$$

Note that the weight $\mu$ is used to divide the set $\pi_{\mathbb{C}} P$ of possible poles of the Mellin symbols into two parts, corresponding to the left resp. the right boundary of $X_{b}^{2}$.

Proposition 5.10. For any $h \in M_{P, w}^{-\infty}\left(\partial X, \Omega^{\frac{1}{2}}\right)$ and any two cut-off functions $\omega_{0}, \omega_{1} \in \mathcal{C}_{c}^{\infty}\left(\overline{\mathbb{R}}_{+}\right)$we have

$$
a:=\varrho^{m} \omega_{0} x^{-m+\ell_{0}} \operatorname{op}_{M}^{\left(\frac{1}{2}-\mu\right)}(h) \omega_{1} \in \widetilde{\Psi}_{b}^{-\infty, \mathcal{E}}\left(X,{ }^{b} \Omega^{\frac{1}{2}}\right) .
$$

Proof. Near $\partial X \times \partial X$ the Schwartz kernel of $a$ is given by

$$
k_{a}=\omega_{0}(x) \omega_{1}\left(x^{\prime}\right) x^{\ell_{0}} \int_{\mathbb{R}_{\xi}}\left(\frac{x}{x^{\prime}}\right)^{-(\mu+i \xi)} h(\mu+i \xi) d \xi\left|\frac{d x}{x} \frac{d x^{\prime}}{x^{\prime}}\right|^{\frac{1}{2}} ;
$$


thus, we obtain for the lifted kernel $\kappa_{a}$ with respect to the singular coordinates $\tau=\frac{x-x^{\prime}}{x+x^{\prime}}$ and $r=x+x^{\prime}$ up to the density factor $\left|\frac{d r}{r} \frac{d \tau}{1-\tau^{2}}\right|^{\frac{1}{2}}$

$$
\kappa_{a}=2 \omega_{0}\left(\frac{r}{2}(1+\tau)\right) \omega_{1}\left(\frac{r}{2}(1-\tau)\right)\left(\frac{r}{2}(1+\tau)\right)^{\ell_{0}} \int_{\mathbb{R}_{\xi}}\left(\frac{1+\tau}{1-\tau}\right)^{-(\mu+i \xi)} h(\mu+i \xi) d \xi .
$$

By the meromorphy of $H_{\tau}: z \longmapsto\left(\frac{1+\tau}{1-\tau}\right)^{-z} h(z) \in \Psi^{-\infty}\left(Y, \Omega^{\frac{1}{2}}\right)$ for $|\tau|<1$ we get for each $N \geq 0$ with $\pi_{\mathbb{C}} P \cap \Gamma_{\mu \pm N}=\emptyset$ :

$$
\int_{\Gamma_{\mu}} H_{\tau}(z) d z=\int_{\Gamma_{\mu \pm N}} H_{\tau}(z) d z \mp 2 \pi i \sum \operatorname{res}_{p} H_{\tau},
$$

where the sum is over all poles $p$ of $H_{\tau}$ with $\operatorname{Re}(p)$ between $\mu$ and $\mu \pm N$. The residues $\operatorname{res}_{p} H_{\tau}$ are given by

$$
\operatorname{res}_{p} H_{\tau}=\left(\frac{1+\tau}{1-\tau}\right)^{-p} \sum_{k=0}^{n_{p}} \frac{(-1)^{k}}{k !} h_{k, p} \log ^{k}\left(\frac{1+\tau}{1-\tau}\right),
$$

where $h_{k, p} \in \Psi^{-\infty}\left(\partial X, \Omega^{\frac{1}{2}}\right)$ are the coefficients of $(z-p)^{-(k+1)}$ in the Laurent expansion of $h$ at $p$. On the other hand, we have for any continuous semi-norm $q$ on $\Psi^{-\infty}\left(Y, \Omega^{\frac{1}{2}}\right)=\mathcal{C}^{\infty}\left(Y^{2}, \Omega^{\frac{1}{2}}\right)$

$$
q\left(\int_{\Gamma_{\mu \pm N}} H_{\tau}(z) d z\right) \leq \operatorname{Const}\left(\frac{1+\tau}{1-\tau}\right)^{-\mu \mp N} .
$$

Because of $\mathrm{lb}=\{\tau=-1\}$ and $\mathrm{rb}=\{\tau=+1\}$, a combination of (5.9), (5.10) and (5.11) completes the proof.

Remark 5.11. The decomposition $U \cong \overline{\mathbb{R}}_{+} \times \partial X$ of $X$ near the boundary induces a tensor decomposition

$$
\mathcal{C}_{c}^{\infty}\left(\left(\beta_{b}^{2}\right)^{-1}(U \times U),{ }^{b} \Omega^{\frac{1}{2}}\right)=\mathcal{C}_{c}^{\infty}\left([-1,1] \times \overline{\mathbb{R}}_{+},\left|\frac{d r}{r} \frac{d \tau}{1-\tau^{2}}\right|^{\frac{1}{2}}\right) \widehat{\otimes}_{\pi} \mathcal{C}^{\infty}\left(Y^{2}, \Omega^{\frac{1}{2}}\right) .
$$

If $P=\left\{\left(p, n_{p}, N_{p}\right): p \in \pi_{\mathbb{C}} P\right\}$ is an asymptotic type, and $h \in M_{P}^{-\infty}\left(\partial X, \Omega^{\frac{1}{2}}\right)$, then the coefficients of $\varrho^{-p+\ell_{0}+i} \log ^{k} \varrho$ resp. $\varrho^{p+i} \log ^{k} \varrho$ in the asymptotic expansion of $\kappa_{a}$ at lb resp. rb belong to $\mathcal{C}_{c}^{\infty}\left([-1,1] \times \overline{\mathbb{R}}_{+},\left|\frac{d r}{r} \frac{d \tau}{1-\tau^{2}}\right|^{\frac{1}{2}}\right) \widehat{\otimes}_{\pi} N_{p}$, by (5.10).

Corolla Ry 5.12. Let $M \in \mathfrak{C}_{w}^{m-j}\left(X,{ }^{b} \Omega^{\frac{1}{2}} ;(\gamma, \gamma-m, k)\right)$ be a smoothing Mellin operator as in Definition 3.8 Then there exists an index family $\mathcal{E}=\left(E_{\mathrm{lb}}, E_{\mathrm{rb}}, 0\right)$ for $X_{b}^{2}$ with $M \in \varrho^{j-m} \widetilde{\Psi}_{b}^{-\infty, \mathcal{E}}\left(X,{ }^{b} \Omega^{\frac{1}{2}}\right)$, and (5.6)

Proof. This is just a combination of (4.3) and Proposition 5.10.

We are now going to consider the other inclusion. Let $\gamma, m \in \mathbb{R}$ and $R \in \mathbb{N}$ be arbitrary. We start with the following observation. 
Lemma 5.13. Let $a \in \widetilde{\Psi}_{b}^{-\infty, \mathcal{E}}\left(X,{ }^{b} \Omega^{\frac{1}{2}}\right)$ be arbitrary.

1. If $\mathcal{E}=(\emptyset, E, 0)$ with a $\mathcal{C}^{\infty}$-index set $E$ satisfying inf $E>\frac{n}{2}-\gamma$, then we have

$$
\varrho^{R-m} a \in \mathfrak{C}_{G, w}\left(X,{ }^{b} \Omega^{\frac{1}{2}},(\gamma, \gamma-m, R)\right)_{O_{R}, Q},
$$

where $Q \in \operatorname{As}_{w}(-\gamma, R)$ is the set of all pairs $(n-z, k)$ with $(z, k) \in E$, $\operatorname{Re}(z)<\frac{n}{2}-\gamma+R$, and $\left(z, k^{\prime}\right) \in E$ only for $k^{\prime} \leq k$.

2. If $\mathcal{E}=(F, \emptyset, 0)$ with a $\mathcal{C}^{\infty}$-index set $F$ satisfying inf $F>\gamma-\frac{n}{2}$, then we have

$$
\varrho^{-m} a \varrho^{R} \in \mathfrak{C}_{G, w}\left(X,{ }^{b} \Omega^{\frac{1}{2}},(\gamma, \gamma-m, R)\right)_{Q, O_{R}},
$$

where $Q \in \operatorname{As}_{w}(\gamma-m, R)$ is the set of all $(m-z, k)$ with $(z, k) \in F$, $\operatorname{Re}(z)<\gamma-\frac{n}{2}+R$, and $\left(z, k^{\prime}\right) \in F$ only if $k^{\prime} \leq k$.

Proof. By (4.8), $g:=\varrho^{R-m} a: \mathcal{H}^{s, \gamma}\left(X,{ }^{b} \Omega^{\frac{1}{2}}\right) \longrightarrow \mathcal{H}^{s^{\prime}, \gamma-m+R}\left(X,{ }^{b} \Omega^{\frac{1}{2}}\right)$ for all $s, s^{\prime} \in \mathbb{R}$, i.e. we have $g: \mathcal{H}^{s, \gamma}\left(X,{ }^{b} \Omega^{\frac{1}{2}}\right) \longrightarrow \mathcal{C}_{\gamma-m, O_{R}}\left(X,{ }^{b} \Omega^{\frac{1}{2}}\right)$; for the adjoint $g^{*}$, note that the terms in the asymptotic expansion of the kernel of $a^{*}$ at lb with $\operatorname{Re}(z)<\frac{n}{2}-\gamma+R$ lead to the terms described by $Q$ whereas, by a result similar to $(4.8)$, the remainders satisfy $\mathcal{H}^{s, m-\gamma}\left(X,{ }^{b} \Omega^{\frac{1}{2}}\right) \longrightarrow \mathcal{H}^{s^{\prime},-\gamma+R-\varepsilon}\left(X,{ }^{b} \Omega^{\frac{1}{2}}\right)$ for all $\varepsilon>0$; thus, $g^{*}: \mathcal{H}^{s, m-\gamma}\left(X,{ }^{b} \Omega^{\frac{1}{2}}\right) \longrightarrow \mathcal{C}_{-\gamma, Q}^{\infty}\left(X,{ }^{b} \Omega^{\frac{1}{2}}\right)$. Finally, (b) follows either by a similar computation or by duality from (a). with

For any $j \in \mathbb{N}_{0}$ with $j<R$, and any index family $\mathcal{E}=\left(E_{\mathrm{lb}}, E_{\mathrm{rb}}, 0\right)$ for $X_{b}^{2}$

$$
-\inf E_{\mathrm{rb}}<\gamma-\frac{n}{2}<\inf E_{\mathrm{lb}}+j
$$

let us denote by $P_{\ell}, \ell=0,1, \ldots, R-j-1$ the weak asymptotic type for Mellin symbols given by

$$
\begin{array}{r}
P_{\ell}:=\left\{(-z+\ell, k):(z, k) \in E_{\mathrm{lb}},\left(z, k^{\prime}\right) \in E_{\mathrm{lb}} \Longrightarrow k^{\prime} \leq k\right\} \\
\cup\left\{(z, k) \in E_{\mathrm{rb}}:\left(z, k^{\prime}\right) \in E_{\mathrm{rb}} \Longrightarrow k^{\prime} \leq k\right\},
\end{array}
$$

and choose $\gamma_{\ell} \in \mathbb{R}$ with $\gamma-\ell-j \leq \gamma_{\ell} \leq \gamma$, and $\pi_{\mathbb{C}} P_{\ell} \cap \Gamma_{\frac{n}{2}-\gamma_{\ell}}=\emptyset$.

Proposition 5.14. For any $a \in \widetilde{\Psi}_{b}^{-\infty, \mathcal{E}}\left(X,{ }^{b} \Omega^{\frac{1}{2}}\right)$, there exist cut-off functions $\omega_{0}, \omega_{1} \in \mathcal{C}_{c}^{\infty}\left(\overline{\mathbb{R}}_{+}\right), h_{\ell} \in M_{P_{\ell}, w}^{-\infty}\left(\partial X, \Omega^{\frac{1}{2}}\right)$, and $G \in \mathfrak{C}_{G, w}\left(X,{ }^{b} \Omega^{\frac{1}{2}},(\gamma, \gamma-m, R)\right)$ such that

$$
\varrho^{j-m} a=\omega_{0}\left(\sum_{\ell=0}^{R-j-1} x^{j-m+\ell} \operatorname{op}_{M}^{\left(\gamma_{\ell}-\frac{n-1}{2}\right)}\left(h_{\ell}\right)\right) \omega_{1}+G .
$$

Proof. By a partition of unity, it suffices to consider the following cases

$$
\begin{aligned}
\operatorname{supp} \kappa_{a} \cap \mathrm{ff}^{b} & =\emptyset, \\
\operatorname{supp} \kappa_{a} \cap \mathrm{lb} & =\emptyset, \text { and } \\
\operatorname{supp} \kappa_{a} \cap \mathrm{rb} & =\emptyset .
\end{aligned}
$$


In case (5.13) we have $a \in \Psi^{-\infty, \mathcal{E}}\left(X,{ }^{b} \Omega^{\frac{1}{2}}\right)$, thus, (5.5) applies, and we obtain $\varrho^{j-m} a \in \mathfrak{C}_{G, w}\left(X,{ }^{b} \Omega^{\frac{1}{2}},(\gamma, \gamma-m, R)\right)$. Therefore, we can assume that there are cut-off functions $\omega_{0}, \omega_{1} \in \mathcal{C}_{c}^{\infty}\left(\overline{\mathbb{R}}_{+}\right)$with $\omega_{0} a \omega_{1}=a$.

In case (5.14), we use the projective coordinates $t=\frac{x^{\prime}}{x}, x, y, y^{\prime}$ near $\mathrm{rb} \cap \mathrm{ff}^{b}$; then there exist $f_{z, k} \in \mathcal{C}_{c}^{\infty}\left(\overline{\mathbb{R}}_{+}\right) \hat{\otimes}_{\pi} \mathcal{C}_{c}^{\infty}\left(\overline{\mathbb{R}}_{+}\right) \hat{\otimes}_{\pi} \mathcal{C}^{\infty}\left(Y^{2}, \Omega^{\frac{1}{2}}\right)$ with

$$
\kappa_{a}-\sum_{(z, k) \in E_{\mathrm{rb},}, \operatorname{Re}(z) \leq N} f_{z, k} t^{z} \log ^{k} t\left|\frac{d t}{t} \frac{d x}{x}\right|^{\frac{1}{2}} \in \dot{\mathcal{C}}_{t}^{N}\left(\overline{\mathbb{R}}_{+}\right) \hat{\otimes}_{\pi} \mathcal{C}_{c}^{\infty}\left(\overline{\mathbb{R}}_{+}\right) \hat{\otimes}_{\pi} \mathcal{C}^{\infty}\left(Y^{2}, \Omega^{\frac{1}{2}}\right)
$$

for all $N \in \mathbb{N}_{0}$. Here $\dot{\mathcal{C}}_{t}^{N}\left(\overline{\mathbb{R}}_{+}\right)$denotes the space of all $N$-times differentiable, compactly supported functions that vanish with all derivatives up to order $N$ at $t=0$. Since $\kappa_{a}$ is smooth up to the front face $\mathrm{ff}^{b}=\{x=0\}$, Taylor expansion with respect to $x$ yields

$$
\begin{aligned}
\kappa_{a}\left(t, x, y, y^{\prime}\right)= & \sum_{\ell=0}^{R-j-1} \underbrace{\frac{1}{\ell !}\left(\partial_{x}^{\ell} \kappa_{a}\right)\left(t, 0, y, y^{\prime}\right)}_{=: \hat{\kappa}_{\ell}\left(t, y, y^{\prime}\right)\left|\frac{d t}{t} \frac{d x}{x} d y d y^{\prime}\right|^{\frac{1}{2}}} x^{\ell} \\
& +\underbrace{\frac{1}{(R-j-1) !} \int_{0}^{1}(1-s)^{R-j-1}\left(\partial_{x}^{R} \kappa_{a}\right)\left(t, s x, y, y^{\prime}\right) d s}_{=: \kappa_{R}\left(t, x, y, y^{\prime}\right)} x^{R-j} .
\end{aligned}
$$

Let $a_{\ell}, \ell=0,1, \ldots, R-j-1$, be the operator corresponding to the kernel

$$
\left(t, x, y, y^{\prime}\right) \longmapsto \omega_{0}(x) \omega_{1}(t x) x^{\ell} \widehat{\kappa}_{\ell}\left(t, y, y^{\prime}\right)\left|\frac{d t}{t} \frac{d x}{x} d y d y^{\prime}\right|^{\frac{1}{2}} .
$$

By differentiating (5.16) with respect to $x$ we get for $\ell=0,1, \ldots, R-j-1$

$$
\left(\tau, y, y^{\prime}\right) \longmapsto \widehat{\kappa}_{\ell}\left(\frac{1+\tau}{1-\tau}, y, y^{\prime}\right)\left|\frac{d \tau}{1-\tau^{2}} d y d y^{\prime}\right|^{\frac{1}{2}} \in \mathcal{A}^{\mathcal{E}_{r}}\left([-1,1] \times Y^{2},{ }^{b} \Omega^{\frac{1}{2}}\right)
$$

with $\mathcal{E}_{r}\left(\{-1\} \times(\partial X)^{2}\right)=E_{\mathrm{rb}}$ and $\mathcal{E}_{r}\left(\{1\} \times(\partial X)^{2}\right)=\emptyset$. Thus, by Proposition 4.5,

$$
\tilde{h}_{\ell}: z \longmapsto \int_{0}^{\infty} t^{z} \hat{\kappa}_{\ell}(t, \cdot, \cdot) \frac{d t}{t} \in \mathcal{C}^{\infty}\left((\partial X)^{2},{ }^{b} \Omega^{\frac{1}{2}}\right)
$$

is meromorphic with poles of order $k+1$ only at those $z$ with $(-z, k) \in E_{\mathrm{rb}}$ and satisfies (4.6), hence $\left[h_{\ell}: z \longmapsto \widetilde{h}_{\ell}(-z)\right] \in M_{P_{\ell}, w}^{-\infty}\left(\partial X, \Omega^{\frac{1}{2}}\right)$, and the Mellin inversion formula gives

$$
\widehat{\kappa}_{\ell}(t)=\int_{\mathbb{R}_{\xi}} t^{\frac{n}{2}-\gamma_{\ell}+i \xi} h_{\ell}\left(\frac{n}{2}-\gamma_{\ell}+i \xi\right) d \xi,
$$

i.e. the Schwartz kernel $k_{\ell}$ of $\varrho^{j-m} a_{\ell}$ is given up to the density factor by

$$
k_{\ell}\left(x, y, x^{\prime}, y^{\prime}\right)=x^{j-m} \omega_{0}(x) \omega_{1}\left(x^{\prime}\right) x^{\ell} \int_{\mathbb{R}_{\xi}}\left(\frac{x^{\prime}}{x}\right)^{\frac{n}{2}-\gamma_{\ell}+i \xi} h_{\ell}\left(\frac{n}{2}-\gamma_{\ell}+i \xi\right) d \xi,
$$


hence $\varrho^{j-m} a_{\ell}=\omega_{0} x^{j-m+\ell} \operatorname{op}_{M}^{\left(\gamma_{\ell}-\frac{n-1}{2}\right)}\left(h_{\ell}\right) \omega_{1}$ for $\ell=0,1, \ldots, R-j-1$. It remains to consider $\varrho^{j-m} \varrho^{R-j} a_{R}=\varrho^{R-m} a_{R}$, where $a_{R} \in \widetilde{\Psi}_{b}^{-\infty,\left(\emptyset, E_{\mathrm{rb},}, 0\right)}\left(X,{ }^{b} \Omega^{\frac{1}{2}}\right)$ corresponds to the kernel

$$
\left(t, x, y, y^{\prime}\right) \longmapsto \omega_{0}(x) \omega_{1}(t x) \kappa_{R}\left(t, x, y, y^{\prime}\right) .
$$

Because of (5.12), Lemma 5.13 gives $\varrho^{-m+R} a_{R} \in \mathfrak{C}_{G, w}\left(X,{ }^{b} \Omega^{\frac{1}{2}},(\gamma, \gamma-m, R)\right)$.

If $\kappa_{a}$ satisfies (5.14), we use the projective coordinates $s=\frac{x}{x^{\prime}}, x^{\prime}, y, y^{\prime}$ near $\mathrm{lb} \cap \mathrm{ff}^{b}$. As above, a Taylor expansion with respect to $x$ yields

$$
\begin{aligned}
\kappa_{a}\left(s, x^{\prime}, y, y^{\prime}\right)= & \sum_{\ell=0}^{R-j-1} \underbrace{\frac{1}{\ell^{\prime}}\left(\partial_{x^{\prime}}^{\ell} \kappa_{a}\right)\left(s, 0, y, y^{\prime}\right)}_{=: \hat{\kappa}_{\ell}\left(s, y, y^{\prime}\right)\left|\frac{d s}{s} \frac{d x^{\prime}}{x^{\prime}} d y d y^{\prime}\right|^{\frac{1}{2}}}\left(x^{\prime}\right)^{\ell} \\
& +\underbrace{\frac{1}{(R-j-1) !} \int_{0}^{1}(1-t)^{R-j-1}\left(\partial_{x}^{R} \kappa_{a}\right)\left(s, t x^{\prime}, y, y^{\prime}\right) d t}_{=\kappa_{R}\left(s, x^{\prime}, y, y^{\prime}\right)}\left(x^{\prime}\right)^{R-j} .
\end{aligned}
$$

By Proposition 4.5, up to a density factor, the Mellin transform $\tilde{h}_{\ell}$ of

$$
\left(\tau, y, y^{\prime}\right) \longmapsto \widehat{\kappa}_{\ell}\left(\frac{1+\tau}{1-\tau}, y, y^{\prime}\right)\left|\frac{d \tau}{1-\tau^{2}} d y d y^{\prime}\right|^{\frac{1}{2}} \in \mathcal{A}^{\mathcal{E}_{l}}\left([-1,1] \times(\partial X)^{2},{ }^{b} \Omega^{\frac{1}{2}}\right)
$$

with $\mathcal{E}_{l}\left(\{-1\} \times(\partial X)^{2}\right)=E_{\mathrm{lb}}$ and $\mathcal{E}_{l}\left(\{1\} \times(\partial X)^{2}\right)=\emptyset$ is meromorphic, has poles of order $k+1$ at all those $z$ with $(-z, k) \in E_{\mathrm{lb}}$, and satisfies (4.6); thus, $\left[h_{\ell}: z \longmapsto \widetilde{h}_{\ell}(z-\ell)\right] \in M_{P_{\ell}, w}^{-\infty}\left(\partial X, \Omega^{\frac{1}{2}}\right)$. Let $a_{\ell}$ be the operator corresponding to the kernel $\left(s, x^{\prime}, y, y^{\prime}\right) \longmapsto \omega_{0}\left(s x^{\prime}\right) \omega_{1}\left(x^{\prime}\right)\left(x^{\prime}\right)^{\ell} \widehat{\kappa}_{\ell}\left(s, y, y^{\prime}\right)\left|\frac{d s}{s} \frac{d x^{\prime}}{x^{\prime}} d y d y^{\prime}\right|^{\frac{1}{2}}$. Using the Mellin inversion formula, we obtain for the Schwartz kernel $k_{\ell}$ of $\varrho^{j-m} a_{\ell}$

$$
\begin{aligned}
& k_{\ell}\left(x, y, x^{\prime}, y^{\prime}\right) \\
= & x^{j-m} \omega_{0}(x) \omega_{1}\left(x^{\prime}\right)\left(x^{\prime}\right)^{\ell} \int_{\mathbb{R}_{\xi}}\left(\frac{x^{\prime}}{x}\right)^{\frac{n}{2}-\gamma_{\ell}-\ell+i \xi} h_{\ell}\left(\frac{n}{2}-\gamma_{\ell}+i \xi, y, y^{\prime}\right) d \xi \\
= & x^{j-m+\ell} \omega_{0}(x) \omega_{1}\left(x^{\prime}\right) \int_{\mathbb{R}_{\xi}}\left(\frac{x^{\prime}}{x}\right)^{\frac{n}{2}-\gamma_{\ell}+i \xi} h_{\ell}\left(\frac{n}{2}-\gamma_{\ell}+i \xi, y, y^{\prime}\right) d \xi,
\end{aligned}
$$

i.e. $\varrho^{j-m} a_{\ell}=\omega_{0} x^{j-m+\ell} \mathrm{op}_{M}^{\left(\gamma_{\ell}-\frac{n-1}{2}\right)}\left(h_{\ell}\right) \omega_{1}$. Let $a_{R} \in \widetilde{\Psi}_{b}^{-\infty,\left(E_{1 \mathrm{l}}, \emptyset, 0\right)}\left(X,{ }^{b} \Omega^{\frac{1}{2}}\right)$ be the operator corresponding to the kernel

$$
\left[\left(s, x, y, y^{\prime}\right) \longmapsto \omega_{0}\left(s x^{\prime}\right) \omega_{1}\left(x^{\prime}\right) \kappa_{R}\left(s, x^{\prime}, y, y^{\prime}\right)\right] \in \mathcal{A}^{\left(E_{1 \mathrm{~b}}, \emptyset, 0\right)}\left(X_{b}^{2},{ }^{b} \Omega^{\frac{1}{2}}\right),
$$

then the remaining part $\varrho^{j}\left(\varrho^{-m} a \varrho^{R}\right) \varrho^{-j}$ belongs to $\mathfrak{C}_{G, w}\left(X,{ }^{b} \Omega^{\frac{1}{2}},(\gamma, \gamma-m, R)\right)$ by [57, Remark 1.2.11] and Lemma 5.13. This completes the proof.

Corollary 5.15. For any index family $\mathcal{E}=\left(E_{\mathrm{lb}}, E_{\mathrm{rb}}, 0\right)$ with $(5.12)$ we have for each $j \in \mathbb{N}_{0}$

$$
\varrho^{j-m} \widetilde{\Psi}_{b}^{-\infty, \mathcal{E}}\left(X,{ }^{b} \Omega^{\frac{1}{2}}\right) \subseteq \mathfrak{C}_{M+G, w}^{m-j}\left(X,{ }^{b} \Omega^{\frac{1}{2}},(\gamma, \gamma-m, \infty)\right) .
$$


The results of this subsection can be summarized as follows.

Theorem 5.16. Let $\gamma, m \in \mathbb{R}$, and $j \in \mathbb{N}_{0}$ be arbitrary. Then we have $\mathfrak{C}_{M+G, w}^{m-j}\left(X,{ }^{b} \Omega^{\frac{1}{2}},(\gamma, \gamma-m, \infty)\right)=\bigcup_{\mathcal{E}} \varrho^{j-m}\left(\widetilde{\Psi}_{b}^{-\infty, \mathcal{E}}\left(X,{ }^{b} \Omega^{\frac{1}{2}}\right)+\Psi^{-\infty, \mathcal{E}}\left(X,{ }^{b} \Omega^{\frac{1}{2}}\right)\right)$, where the union is over all index families $\mathcal{E}=\left(E_{\mathrm{lb}}, E_{\mathrm{rb}}, 0\right)$ satisfying $(5.12)$.

Proof. This is just a combination of Theorem 5.8, Corollary 5.12, Corollary 5.15 , and Subsection 5.3.

5.5. Summary. A combination of the results of the previous subsections now leads to the main result of this paper.

Theorem 5.17. Let $\gamma, m \in \mathbb{R}, j \in \mathbb{N}_{0}$ be arbitrary, and suppose that the boundary $\partial X$ of $X$ is connected. Then we have

$$
\begin{aligned}
\mathfrak{C}^{m-j}\left(X,{ }^{b} \Omega^{\frac{1}{2}},(\gamma, \gamma-m, \infty)\right) & \left.\subset \bigcup_{\mathcal{E}} \varrho^{j-m} \Psi_{b, c l}^{m-j, \mathcal{E}}\left(X,{ }^{b} \Omega^{\frac{1}{2}}\right)\right) \\
& =\mathfrak{C}_{w}^{m-j}\left(X,{ }^{b} \Omega^{\frac{1}{2}},(\gamma, \gamma-m, \infty)\right),
\end{aligned}
$$

where the union is over all index families $\mathcal{E}=\left(E_{\mathrm{lb}}, E_{\mathrm{rb}}, 0\right)$ satisfying

$$
-\inf E_{\mathrm{rb}}<\gamma-\frac{n}{2}<\inf E_{\mathrm{lb}}+j
$$

Proof. The theorem follows immediately from Theorem 5.4, Theorem 5.8, and Theorem 5.16.

Roughly speaking, the main difference between the cone algebra and the full bcalculus is the additional asymptotic information encoded in the finite-dimensional subspaces describing the Laurent coefficients of the corresponding meromorphic functions. Anyway, it is straightforward to include these additional symptotic data into the definition of the full b-calculus as indicated in Remark 5.11; however, note that this requires as in the definition of the cone algebra a non-natural choice of a product decomposition near the boundary. This "strong" version of the b-calculus coincides then with the cone algebra.

For manifolds with disconnected boundary it has been shown in Theorem 5.4 that a holomorphic version of the cone algebra coincides with the small overblown b-calculus. An analogue of Theorem 5.17 remains true provided we replace the full b-calculus $\Psi_{b, c l}^{m-j, \mathcal{E}}\left(X,{ }^{b} \Omega^{\frac{1}{2}}\right)$ by the full overblown b-calculus. The details are left to the reader.

\section{References}

[1] J. Brüning. Index theory for regular singular operators and applications. In Proc. Oberwolfach 1986, Lect. Notes in Math. 1256, Springer Verlag, Berlin, 1987.

[2] J. Brüning, R. T. Seeley. An index theorem for first order regular singular operators. Amer. J. Math. 110: 659-714, 1988

[3] J. Cheeger. Spectral geometry of singular Riemannian spaces. J. Differ. Geom. 18: 575-657, 1983.

[4] Y. V. Egorov, B.-W. Schulze. Pseudo-differential Operators, Singularities, Applications. Birkhäuser, Basel, 1997. 
[5] C. L. Epstein, R. B. Melrose, G. A. Mendoza. Resolvent of the Laplacian on strictly pseudoconvex domains. Acta Math. 167: 1-106, 1991.

[6] B. V. Fedosov, B.-W. Schulze. On the index of elliptic operators on a cone. In M. Demuth et al. (eds.), Schrödinger Operators, Markov Semigroups, Wavelet Analysis, Operator Algebras, Math. Topics, volume 14: Advances in Part. Diff. Equ., Akademie Verlag, Berlin, 1996

[7] B. V. Fedosov, B.-W. Schulze, N. Tarkhanov. On the index of elliptic operators on a wedge. J. Funct. Anal. 157: 164-209, 1998.

[8] B. Gramsch. Relative Inversion in der Störungstheorie von Operatoren und $\Psi$-Algebren Math. Ann. 269: 27-71, 1984.

[9] B. Gramsch, J. Ueberberg, K. Wagner. Spectral invariance and submultiplicativity for Fréchet algebras with applications to pseudo-differential operators and $\Psi^{*}$-quantization. In Operator Theory: Advances and Applications, volume 57, Birkhäuser, Basel, 1992.

[10] L. Hörmander. Fourier integral operators I. Acta Math. 127: 79-183, 1971.

[11] L. Hörmander. The analysis of linear partial differential operators, volume 3, SpringerVerlag, Berlin, 1985.

[12] D. Kapanadze, B.-W. Schulze, I. Witt. Coordinate invariance of the cone algebra with asymptotics. (in preparation)

[13] V. A. Kondrat'ev. Boundary value problems for elliptic equations in domains with conical or angular points. Trans. Mosc. Math. Soc. 16: 227-313, 1967.

[14] R. Lauter. An operator theoretical approach to enveloping $\Psi^{*}$ - and $C^{*}$-algebras of Melrose algebras of totally characteristic pseudodifferential operators. Math. Nachr. 196: 141-166, 1998.

[15] R. Lauter. Holomorphic functional calculus in several variables and $\Psi^{*}$-algebras of totally characteristic operators on manifolds with boundary. Shaker Verlag, Aachen, 1997. 241 pages.

[16] R. Lauter. On $\Psi^{*}$ - and $C^{*}$-algebras of pseudodifferential operators on manifolds with conical singularities. In E. Albrecht et al. (eds.), Banach algebras. Proceedings of the 13th international conference on Banach algebras, University of Tuebingen, Blaubeuren (Germany), July 20 - August 3, 1997. Walter de Gruyter, Berlin 1998.

[17] R. Lauter. O predstavleniyah $\Psi^{*}-\mathbf{i} C^{*}$ - algebr psevdodifferentsial'nyh operatorov na mnogoobraziyah s vershinami. In N. N. Ural'tseva (ed.), Nelineinye uravneniya s chastnymi proizvodnymi i teoriya funktsii, Problemy matematicheskogo analiza, volume 18. SanktPeterburgskii universitet, 1998. (in Russian).

[18] R. Lauter. On the existence and structure of $\Psi^{*}$-algebras of totally characteristic operators on compact manifolds with boundary. Preprint 12/97, Fachbereich Mathematik, Johannes Gutenberg-Universität Mainz, 1997. (to appear in J. Funct. Anal.).

[19] R. Lauter. The length of $C^{*}$-algebras of b-pseudodifferential operators. Preprint $12 / 98$ Fachbereich Mathematik, Johannes Gutenberg-Universität Mainz, 1998. (to appear in Proc. Amer. Math. Soc.).

[20] R. Lauter, S. Moroianu. Fredholm theory for degenerate pseudodifferential operators on manifolds with fibred boundaries. (to appear in: Comm. Partial Differ. Equations).

[21] M. Lesch. Operators of Fuchs type, conical singularities, and asymptotic methods. TeubnerTexte zur Mathematik 136. Teubner-Verlag, Stuttgart, 1997.

[22] P. A. Loya. On the b-pseudodifferential calculus on manifolds with corners. PhD-thesis, Massachusetts Institute of Technology, Cambridge, 1998

[23] F. Mantlik. Norm closure and extension of the symbolic calculus for the cone algebra. Ann. Global Anal. Geom. 13:339-376, 1995.

[24] F. Mantlik. Norm closure of operator algebras with symbolic structure. Math. Nachr. 201: 91-116, 1999

[25] R. R. Mazzeo. The Hodge cohomology of a conformally compact metric. J. Differ. Geom. 28: 309-339, 1988 
[26] R. R. Mazzeo. Elliptic theory of differential edge operators I. Comm. Partial Differ. Equations 16: 1615-1664, 1991.

[27] R. R. Mazzeo, R. B. Melrose. Analytic surgery and the eta invariant. Geom. Funct. Anal. 5: $14-75,1995$.

[28] R. B. Melrose. Transformation of boundary value problems. Acta Math. 147: 149-236, 1981.

[29] R. B. Melrose. Pseudodifferential operators, corners and singular limits. Proceedings of the International Congress of Mathematicians held in Kyoto (Japan), Springer Verlag, Berlin, 1990.

[30] R. B. Melrose. Calculus of conormal distributions on manifolds with corners. Int. Math. Res. Not. 3: 51-61, 1992.

[31] R. B. Melrose. The Atiyah-Patodi-Singer Index Theorem, Res. Notes in Math., volume 4, A K Peters, Wellesley, Massachusetts, 1993.

[32] R. B. Melrose. Spectral and scattering theory for the Laplacian on asymptotically Euclidean space. In M. Ikawa (ed.), Spectral and Scattering Theory, Proceedings of the Taniguchi International Workshop held in Sanda (Japan), November 1992. Lect. Notes Pure Appl. Math., Marcel Dekker Inc., New York, 1994.

[33] R. B. Melrose. Geometric Scattering Theory. Cambridge University Press, 1995.

[34] R. B. Melrose. Geometric optics and the bottom of the spectrum. In F. Colombini et al. (eds.), Geometrical optics and related topics, Progress in nonlinear differential equations and their applications, volume 32, Birkhäuser, Basel, 1997.

[35] R. B. Melrose. Analysis on Manifolds with Corners. (in preparation).

[36] R. B. Melrose, G. Mendoza. Elliptic operators of totally characteristic type. MSRI Preprint, 1983.

[37] R. B. Melrose, V. Nistor. K-theory of $C^{*}$-algebras of b-pseudodifferential operators. Geom. Funct. Anal. 8: 88-122, 1998.

[38] R. B. Melrose, V. Nistor. Homology of pseudodifferential operators I. Manifolds with boundary. Preprint, May 1996. (to appear in: Amer. Math. J.).

[39] R. B. Melrose, P. Piazza. Analytic K-theory on manifolds with corners. Adv. Math. 92: 1-26, 1992.

[40] V. E. Nazaikinskii, B.-W. Schulze, B. Y. Sternin. On the homotopy classification of elliptic operators on manifolds with singularities. Preprint 99/21, University of Potsdam, 1999.

[41] P. Piazza. On the index of elliptic operators on manifolds with boundary. J. Funct. Anal. 117: 308-359, 1993.

[42] B. A. Plamenevskij. On algebras generated by pseudodifferential operators with isolated singularities in the symbols. Sel. Math. Sov. 5: 77-100, 1986. Originally published in: Problemy Matematicheskoi fiziki, No. 10 Spektralnaya Teoriya, volnovoye protsessy, Leningrad University Press, 1982, pp. 209-241.

[43] B. A. Plamenevskij. Algebras of Pseudodifferential Operators. Kluwer Academic Publishers, Dordrecht, 1989. Originally published in: Nauka, Moscow, 1986.

[44] S. Rempel, B.-W. Schulze. Index Theory of Elliptic Boundary Value Problems. Akademie Verlag, Berlin, 1982.

[45] S. Rempel, B.-W. Schulze. Complete Mellin and Green symbolic calculus in spaces with conormal asymptotics. Ann. Global Anal. Geom. 4: 137-224, 1986.

[46] E. Schrohe. Invariance of the cone calculus without asymptotics. Ann. Global Anal. Geom. 14: 403-425, 1996.

[47] E. Schrohe. Noncommutative residues and manifolds with conical singularities. J. Funct. Anal. 150: 146-174, 1997.

[48] E. Schrohe, B.-W. Schulze. Boundary value problems in Boutet de Monvel's algebra for manifolds with conical singularities I. In M. Demuth et al. (eds.), Pseudo-differential Calculus and Mathematical Physics, Math. Topics, volume 5: Advances in Part. Diff. Equ., Akademie Verlag, Berlin, 1994 
[49] E. Schrohe, B.-W. Schulze. Boundary value problems in Boutet de Monvel's algebra for manifolds with conical singularities II. In M. Demuth et al. (eds.), Boundary Value Problems, Schrödinger Operators, Deformation Quantization, Math. Topics, volume 8: Advances in Part. Diff. Equ., Akademie Verlag, Berlin, 1996.

[50] E. Schrohe, B.-W. Schulze. Mellin and Green symbols for boundary value problems on manifolds with edges. Integral Equations Oper. Theory 34: 339-363, 1999.

[51] E. Schrohe, B.-W. Schulze. Pseudodifferential boundary value problems on manifolds with edges. (in preparation).

[52] E. Schrohe, J. Seiler. Ellipticity and Invertibility in the cone algebra on $L_{p}$-Sobolev spaces. Preprint, Inst. für Math., Potsdam, 1999.

[53] B.-W. Schulze. Mellin Expansions of pseudo-differential operators and conormal asymptotics of solutions. In Proc. Oberwolfach 1986, Lect. Notes in Math. 1256, Springer Verlag, Berlin 1987.

[54] B.-W. Schulze. Pseudo-differential operators on manifolds with edges. In H. Triebel et al. (eds.), Symposium 'Partial Differential Equations' 1988, Holzhau (Germany), Teubner Texte zur Mathematik 112, Teubner-Verlag, Stuttgart, 1989.

[55] B.-W. Schulze. Pseudo-differential Operators on Manifolds with Singularities. North Holland, Amsterdam, 1991.

[56] B.-W. Schulze. The Mellin pseudo-differential calculus on manifolds with corners. In H Triebel et al. (eds.), Symposium 'Analysis on Manifolds with Singularities' 1990, Breitenbrunn (Germany), Teubner Texte zur Math. 131, Teubner-Verlag, Stuttgart, 1992.

[57] B.-W. Schulze. Pseudo-differential Boundary Value Problems, Conical Singularities, and Asymptotics. Math. Topics 4, Akademie Verlag, Berlin, 1994.

[58] B.-W. Schulze. Boundary Value Problems and Singular Pseudo-differential Operators. J. Wiley, Chichseter, 1998.

[59] B.-W. Schulze. Operators on Corner Manifolds. J. Wiley, Chichester, (in preparation).

[60] B.-W. Schulze. Operator algebras with symbol hierarchies on manifolds with singularities. In J. B. Gil et al. (eds.), Approaches to Singular Analysis, Operator Theory: Advances and Applications, Birkhäuser, Basel. (to appear).

[61] J. Seiler. Pseudo-differential calculus on manifolds with non-compact edges. PhD-thesis, Universität Potsdam, 1997.

[62] J. Seiler. Mellin and Green pseudo-differential operators associated with noncompact edges. Integral Equations Oper. Theory 31:214-245, 1998.

[63] J. Seiler. Continuity of edge and corner pseudo-differential operators. Math. Nachr. 205: 163-182, 1999.

[64] J. Seiler. The cone algebra and a kernel characterization of Green operators. In J. B. Gil et al. (eds.), Approaches to Singular Analysis, Operator Theory: Advances and Applications, Birkhäuser, Basel. (to appear).

[65] A. Unterberger. The calculus of pseudo-differential operators of Fuchs type. Comm. Partial Differ. Equations 9: 1179-1236, 1984.

Johannes Gutenberg-Universität Mainz, Fachbereich Mathematik, 55099 Mainz, Germany

E-mail address: lauter@mathematik.uni-mainz.de

Universität Potsdam, Institut für Mathematik, Postfach 6015 53, 14415 Potsdam, GERMANY

E-mail address: seiler@math.uni-potsdam.de 